Open Access

\title{
Characterizing communities of hashtag usage on twitter during the 2020 COVID-19 pandemic by multi-view clustering
}

\author{
lain J. Cruickshank ${ }^{*}$ (D) and Kathleen M. Carley
}

\section{*Correspondence: \\ icruicks@andrew.cmu.edu \\ CASOS, Carnegie Mellon University, 5000 Forbes Ave, Pittsburgh, PA,} USA

\begin{abstract}
The COVID-19 pandemic has produced a flurry of online activity on social media sites. As such, analysis of social media data during the COVID-19 pandemic can produce unique insights into discussion topics and how those topics evolve over the course of the pandemic. In this study, we propose analyzing discussion topics on Twitter by clustering hashtags. In order to obtain high-quality clusters of the Twitter hashtags, we also propose a novel multi-view clustering technique that incorporates multiple different data types that can be used to describe how users interact with hashtags. The results of our multi-view clustering show that there are distinct temporal and topical trends present within COVID-19 twitter discussion. In particular, we find that some topical clusters of hashtags shift over the course of the pandemic, while others are persistent throughout, and that there are distinct temporal trends in hashtag usage. This study is the first to use multi-view clustering to analyze hashtags and the first analysis of the greater trends of discussion occurring online during the COVID-19 pandemic.
\end{abstract}

Keywords: Social media, Clustering, Multi-view data, COVID-19

\section{Introduction}

At the time of the writing of these words, the world is undergoing a pandemic. This pandemic, which is caused by the SARS-CoV-2 virus and often referred to as the COVID-19 pandemic, has caused immense societal and economic disruption across the world. Since the onset of the COVID-19 pandemic many nations have adopted a social-distancing strategy which has had the unintended consequence of emphasizing and increasing the role of social media in linking people together (Article19 2020; Hussain 2020). Consequently, the study of social media data during the current COVID-19 pandemic can provide unique insights into online social behavior.

Thus far, much of the work with COVID-19 social media data has focused on the prevalence and spread of COVID-19 misinformation. There has been less work on understanding what are the important topics of discussion associated with the pandemic and how those discussion topics may change over the course of the pandemic. One social media innovation which can be used to characterize and understand topics of social

(C) The Author(s). 2020 Open Access This article is licensed under a Creative Commons Attribution 4.0 International License which permits use, sharing, adaptation, distribution and reproduction in any medium or format, as long as you give appropriate credit to the original author(s) and the source, provide a link to the Creative Commons licence, and indicate if changes were made. The images or other third party material in this article are included in the article's Creative Commons licence, unless indicated otherwise in a credit line to the material. If material is not included in the article's Creative Commons licence and your intended use is not permitted by statutory regulation or exceeds the permitted use, you will need to obtain permission directly from the copyright holder. To view a copy of this licence, visit http://creativecommons.org/licenses/by/4.0/. 
media conversations are hashtags. Hashtags are a social media innovation which were designed to allow users to easily find and interact with certain discussion topics. So, in this work, we propose clustering hashtags from COVID-19 social media data in order to understand the topics of discussion happening within the greater COVID-19 social media discussion.

In order to cluster hashtags from social media data, we propose the use of multi-view clustering. Multi-view clustering is the family of techniques that seek to cluster data which has divisions within the data (which could be of different types of data) each of which describe the same set of entities (Baltrušaitis et al. 2019). Multi-view clustering seeks to combine all of the different divisions, or views of the data, in order to produce a better clustering of the data. Many naturally-occurring, social phenomena give rise to multiple types and views of data. So, using a clustering method that can exploit information from all of those views should result in a better clustering of the data. To date and the best of the authors' knowledge, most clustering of social media data is not clustered by multiview clustering. So, in this work, we propose the use of multi-view clustering in order to better understand the discussion topics surrounding COVID-19 on social media. In particular, we analyze topical clusters of twitter data by multi-view clustering on hashtags, where we use the co-occurrence of hashtags, tweet text, twitter users, and Uniform Resource Locators (URLs) that co-occur with hashtags in a tweet as the views to cluster the hashtags.

The main contributions of this work are in twofold. First, we advance the analysis of complex networks through the first use of a multi-view clustering technique on a large, social-based data set; multiple collections of objects, each consisting of upwards of 85,000 objects, are clustered in this study. Whereas, most multi-view clustering techniques have been used on data sets, social-based or otherwise, of at most one collection of 70,000 objects (i.e. full MNIST data set). Second, from the results of multi-view clustering of hashtags, we provide a characterization of topical clusters of hashtags that give distinct insight into what conversations surround the COVID-19 pandemic on twitter. These topical groups of hashtag usage include co-opting of the COVID-19 calamity to support different causes and a persistent coupling of U.S. politics related hashtags with conspiracy theory related hashtags

The article is organized as follows: In the next section we provide some background on COVID-19 social media analyses and multi-view clustering. In the "Data", we provide an overview of the data used in this study and the steps taken to process the data. In the "Methodology" we describe the methodology proposed in this work for clustering multiview, social-based data, like hashtags, and the settings used for this study's data. In the "Results" sections we present the results of the multi-view clustering, identify different time periods of hashtag usage, perform an ensemble clustering of the hashtag clusters to produce prototypical hashtags clusters for the different time periods, and then qualitatively analyze those clusters and the user bases of the clusters. Finally, we conclude the study with a discussion of the results and directions for future work.

\section{Background}

Current study into online social behavior during the COVID-19 pandemic has largely focused on how information and misinformation operates during a pandemic. This is because good information is a key enabler to combat the effects of the pandemic whereas 
misinformation can exacerbate its effects (Hussain 2020; Gallotti et al. 2020). Recent studies into the prevalence and persistence of misinformation have shown that misinformation on the COVID-19 pandemic has been especially persistent and spreads through online social networks quickly (Article19 2020; Boberg et al. 2020; Yang et al. 2020). The spread of COVID-19 misinformation has become so problematic and widespread that many researchers are referring to it as an 'Infodemic' (Article19 2020; Cinelli et al. 2020; Gallotti et al. 2020). The Infodemic is characterized by a virus-like spread of misinformation across many different communication mediums, most notably online social networks. Additionally, other researchers have identified important mechanisms by which the misinformation propagates in social media. Recent research has identified the importance of bots in the spread of misinformation (Ferrara 2020). Other research has highlighted the role of alternative news sources and user characteristics like political beliefs in the spread of COVID-19 misinformation (Boberg et al. 2020; Huang 2020).

One area that is less clear is how social media users may be changing their behavior and how social media communities and discussions are changing in response to the COVID19 pandemic and Infodemic. While the aforementioned research has shown that social media users are spreading COVID-19 misinformation, sometimes even faster than good information, its not clear how users' interactions or how discussion communities may be changing during the pandemic. It is also not clear if there are any topical areas of focus for social media users during the ongoing pandemic. For example, are social media discussions focusing around topics like health and welfare or around politics and business, or any combination thereof?

One of the recent social media innovations that have been used to track and understand conversations and conversational topics are hashtags. Hashtags originated in 2007 on the social media platform Twitter as a means of allowing users to efficiently retrieve information relevant to a topic (Zhang 2019). The use of hashtags on Twitter has expanded to not only be a means of characterizing discussion topics, but also a means of predicting user links and characterizing both communities of users as well as the users themselves (Shapp 2014; Saxton et al. 2015; Sheldon et al. 2019; Xiao et al. 2014; Zhang 2019). As such, clustering of hashtags can be used to understand topics of interest for social media users and the communities that form around certain discussions (Kywe et al. 2012; Vicient and Moreno 2015). The clustering of hashtags has been done by either the text context used with the hashtags, co-occurrence of hashtags within the same social media post (i.e. same tweet), or by having similar users that use the same hashtags (Kywe et al. 2012; Xiao et al. 2014). More recent work on clustering hashtags has focused on better feature engineering of the text that accompanies hashtags in order to capture the semantic meaning of the text and thus better hashtag clusters (Vicient and Moreno 2015). To date, no work has attempted to combine all of these different views of hashtag usage and use multi-view clustering to cluster hashtags in order analyze social media data for understanding topics of interest for social media users.

\section{Multi-view clustering}

Multi-view clustering techniques are techniques designed to handle clustering of objects which can be described by more than one data source. Many different real-world, social phenomena give rise to 'views' of data which are often different types of data that can be used to describe the same set of actors. For example, social media users can post content, 
which could give rise to a text view, and have interactions with each other, which can give rise to network views. Multi-view clustering aims to fuse the information from these different views of the data to produce one clustering of the object that created the data (Baltrušaitis et al. 2019; Baltrusaitis et al. 2017; Ye et al. 2018; Yang and Wang 2018). The advantage of multi-view clustering derives form two basic principles: complementarity and consensus. The complementarity principle is that multiple views of data can be employed to more comprehensively and accurately describe the phenomena of interest (Yang and Wang 2018). With multi-view data, any single view is sufficient for the clustering task, but the combination of multiple views can provide complementary information to any single view which improves the clustering task. The consensus principle is the idea that a clustering solution should be maximally consistent across the multiple views (Yang and Wang 2018). This principle implies that finding a clustering solution that reduces the error between it and all of the view clustering solutions will be the optimal clustering solution. Multi-view clustering techniques also break into the three paradigms of early, intermediate, and late integration techniques (Baltrušaitis et al. 2019). Intermediate integration techniques, which are those which work directly on the multi-view data in a multi-view format to produce the desired output, as typically regarded as the superior paradigm for multi-view clustering.

In terms of applications of multi-view clustering, there have been a surge of new techniques developed in multi-view clustering for handling genetic (Huang et al. 2017; Zitnik et al. 2018) and image data (Bai et al. 2018; Yang and Wang 2018). Many of these techniques are intermediate integration techniques that rely on producing graphs of each view of the data and then collectively clustering those graphs (Baltrušaitis et al. 2019; Baltrusaitis et al. 2017). By doing so, these techniques can then define one function across all of the views for measuring the goodness of the clustering and exploit properties of graphs in modeling and preserving complex structures in the data, as is often done with spectral clustering (Guo et al. 2014; Xia et al. 2014; Xu and Ke 2016; Yu et al. 2017). While these techniques can be used - at least in principle - with any kind of data, very few have been applied to social-based, multi-view data.

Part of the difficulty in multi-view clustering of social media data like hashtags are that the data is often very large (on the order of tens or hundreds of thousands of hashtags being used in any given conversation) which can pose problems for many of the existing multi-view clustering techniques. Additionally, social media data often have partiallycomplete views of data; social-media users and objects, like hashtags, may not have any interactions within some views. For example, a hashtag may never co-occur with another hashtag, or a user may never engage in an activity like re-tweeting. These partially complete views pose challenges for many existing multi-view clustering techniques as these objects naturally become isolates or small connected components in the view graphs used in the clustering.

Finally, Within the realm of social-based data, and in particular social network analysis, there are two main areas of research that deal with multi-view data. The first is multilayer or multiplex social network analysis. Multiplex networks are networks in which a node has more than one type of link connecting it to other nodes (Aleta and Moreno 2019). These networks can be modeled as a collection of networks that are defined over the same nodeset but have different links within each of the networks. So, in this data format, each network represents a possible view of the data. Multi-layer networks contain 
the same networks as multiplex networks, but with the addition of inter-layer links where a node in one layer can be connected to nodes in other layers (Aleta and Moreno 2019). As with multiplex networks, each layer can be considered a view of the data. There have been a host of techniques designed to work with multi-layer and multiplex networks, most of which leverage Network Modularity as the objective function to cluster the networks (Mucha et al. 2010; Pamfil et al. 2018; Tagarelli et al. 2017), or use a stochastic blockmodel (Huang et al. 2019; Ni et al. 2015; Pamfil et al. 2018). So, while there are a wealth of techniques available, these techniques are limited to multi-view data that only consists of networks.

The second main area of multi-view clustering of social-based data are attributed networks. Attributed networks are networks which also have additional information on the nodes (Chunaev 2019). So, an attributed network will have two views of data; one view which is the network itself and a second view of features describing the nodes present in the network. Attributed networks are often clustered by either combining the attribute information into the network itself and then clustering that network by standard network clustering techniques (Alinezhad et al. 2019; Chunaev 2019; Papadopoulos et al. 2015; Papadopoulos et al.2017), or by defining a new measure of network modularity that incorporates a term for the attributes (Combe et al. 2015; Chunaev 2019). So, attributed network clustering is limited to just the multi-view data scenario in which there is one network view and one non-network view of the data.

\section{Data}

The data for this analysis comes from Twitter's streaming API ${ }^{1}$. The data collection was done using a list of key words including "coronavirus", "coronaravirus", "wuhan virus", "wuhanvirus", "2019nCoV", "NCoV", "NCoV2019” (Huang 2020). The collected data spans the time period from 1 February 2020 to 30 April 2020 and consists of over 300 million tweets that have, on average, 45,000 unique hashtags per day. The following figure, Fig. 1 depicts the daily statistics concerning the use of hashtags within the data set. It should be noted that as a prepossessing step, any hashtag that was not used in at least 3 tweets was not included in the data. These hashtags are often misspellings of more widely used hashtags.

Hashtag usage within the data is both prevalent and increases in the diversity of hashtags being used over time. The use of unique hashtags generally increase over the time period of data collection and displays some weekly cyclical patterns as well (i.e. slight drops in the number of unique hashtags being used on weekend days). It is interesting to note that these counts are counts of unique hashtags and not the total use of hashtags. So, it is possible that as the scope of the COVID-19 pandemic expanded, hashtags that were originally unassociated with the COVID-19 pandemic end up becoming a part of the conversation. It is also possible that as the scope of the pandemic expanded, that new hashtags were invented to better address the changing needs of the conversation about the pandemic. Additionally, there is a relatively high ratio of hashtags being used in tweets throughout all of the data (greater than $40 \%$ of tweets have a hashtag). While this is in part due to the means of collecting the data, it also reflects a trend observed by other authors of increasing 


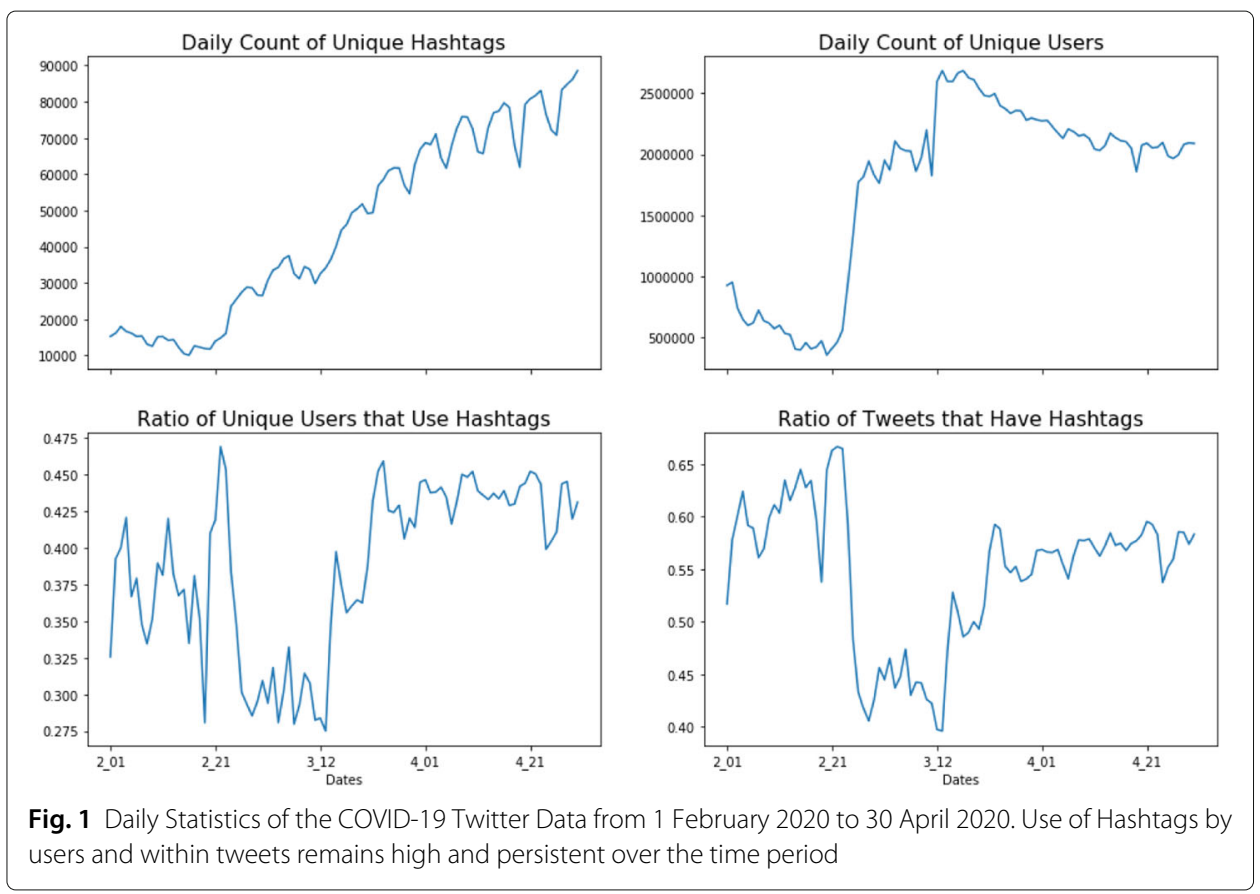

hashtag usage among social media users generally (Sheldon et al. 2019; Zhang 2019). The ratio of hashtag usage in tweets has three observable phases over time. In the first phase, from 1 February to 24 February, the ratio of hashtags present in tweets remains at its highest, with a slight positive trend. Then, starting on the 25th of February the ratio of hashtags in tweets decreases and remains lower until the 15th of March. Finally, in the third phase from the 16th of March until the 30th of April, the ratio of hashtags being used in tweets remains stable at around 55\% of the tweets. So, while there is a high percentage of tweets that have at least one hashtag, this percentage is not stable over time. While its not clear why this temporal trend exists, it is likely related to the dynamic nature of the users interacting on Twitter over the various stages of the COVID-19 pandemic.

There is also a reasonably high percentage of hashtag use among individual users, which generally increases with time. As with the ratio of tweets that feature a hashtag, the ratio of users that use at least one hashtag on a given day breaks into three observable periods. The first period is a period of a lot of oscillation in daily hashtag usage centered around $37 \%$. The second period is a decline in hashtag use corresponding to the same period of decreased hashtags being used in individual tweets, from 25 February to 15 March. The third period is from the 16th of March to the 30th of April and has a sustained hashtag use at around $43 \%$ of users using at least one hashtag in a tweet per day. Overall, 50.47\% of users use at least one hashtag during the three months that data was collected. In terms of individual hashtag usage, for users in general the usage statistics of hashtags are as follows: min: 0, max: $100 \%$, mean: $31.6 \%$, and standard deviation: $38.8 \%$ of their tweets featuring hashtags. Of those users that use at least on hashtag in their tweets, the usage statistics become: min: $1.9 \%$, max: $100 \%$, mean $62.6 \%$, and standard deviation: $32.4 \%$ of their tweets featuring hashtags.

This hashtag usage takes place in a background of variable trends in the number of unique daily users present within the data set. There is a declining number of unique 
users within the data from the 1st of February to around the 21st of February, at which point there is a large increase in the number of unique users until the 20th of March. From the 20th of March to the end of April, the number of daily unique users begins to decline again. So, while the use of hashtags increases as well as the use of unique hashtags increases, the number of unique users actually declines. So, during the early stages of the pandemic there are a fairly small number of users tweeting relevant tweets which often have hashtags which then transitions over the course of the pandemic to a much larger user base that does not initially use many hashtags. This observation suggests that user's interactions on Twitter are dynamic over the course of a pandemic and that discussion topics, in the form of hashtags are also dynamic over the course of a pandemic. It also suggests that hashtag usage becomes more widely adopted after an initial surge in users possibly as means of better characterizing the new and burgeoning conversations happening on Twitter surrounding the pandemic. All together, the nature of the use of hashtags and the hashtags in use have likely changed over the course of the COVID-19 pandemic.

\section{Data processing}

The aforementioned collection of raw tweets was then transformed into view data for clustering of the hashtags. First, the data was separated into days. Second, the daily tweet data was preprocessed as follows: First, any hashtag which was used in less than 3 tweets was excluded from the data. In experimental iterations of clustering the data, we found that those hashtags that were mentioned in very few tweets often did not have enough information in the different views of the data to measure their similarity with other hashtags to meaningfully cluster them. Generally, those hashtags which were used in few tweets were often isolates in most or all of the view graphs. Based on these observations, we empirically found three to be a suitable minimum number of tweets for a hashtag such that hashtag could be effectively clustered. Second, the tweet text was preprocessed by removing all of hashtags, URLs, symbols (i.e. emojis, punctuation, etc.), and twitterspecific tags (i.e. mentions, quotes, etc.) from the tweet text. From there, for each hashtag, the accompanying text, other hashtags, the user, and any URLs used in each tweet that contained that hashtag were extracted. This data was then separated into four separate views of the data. The first view is the text view and contains all of the pre-processed text from each tweet that features a hashtag. The intuition behind this view is that the text accompanying a particular hashtag may give insight into how and what a particular hashtag is used for in discussions. The accompanying tweet text, whether used in a raw form or given semantic enhancement has been previously used to cluster hashtags (Kywe et al. 2012; Vicient and Moreno 2015). The second view is the users which tweet a hashtag with the idea that users may be partial to tweeting particular hashtags as part of a discussion. Shared users have also been used to cluster hashtags in previous works (Kywe et al. 2012). The third view is the URLs which co-occur with a hashtag. Since URLs are often used as information to support claims in tweets, this view should give insight into what information is underlying the use of certain hashtags. Finally, the fourth view is the co-occurrence of hashtags within tweets. Within any given tweet using hashtags, a user may use multiple, related hashtags as part of their tweet. The co-occurrence of hashtags is frequently used to create hashtag-to-hashtag networks for analysis by standard network science techniques (Vicient and Moreno 2015). So, all together, the collected tweets are processed to create four different views of the data for each day in order to cluster the hashtags. 


\section{Methodology}

In this section, the method for performing a multi-view clustering of the hashtags is detailed. As was mentioned in the "Background" section, multi-view clustering of socialbased objects poses some distinct challenges. Namely, there are hundreds of thousands of hashtags that need to be clustered, partially complete views, and the views consist of both network and non-network data. As such, we propose a new method of multiview clustering that can handle all of the aspects of this data. As with any multi-view clustering technique, there are two main requirements: conversion of the views of the data into a format which can fuse information from the views together, and a clustering goodness function that is defined for all of the views used in the clustering. For the first requirement, we adopt the method used in many multi-view clustering techniques of converting all of the views of the data into graphs. This allows for preservation of local structures in the data and provides a flexible format that can be used with any data type (Brugere et al. 2018; Qiao et al. 2018). For the second requirement, we use network modularity due to its effectiveness as a clustering quality function for networks or graphs and because many fast, scalable heuristics already exist for maximizing network modularity (i.e. Louvain (Blondel et al. 2008), Leiden (Traag et al. 2019), etc.). We refer to this multiview clustering technique as Multi-view Modularity Clustering (MVMC). The following figure, Fig. 2, displays the overall methodology.

The methodology proposed in this work for clustering multi-view, social-based data consists of two steps. The first step is to form graphs for every view of the data (A in Fig. 2). So, a view graph has vertices which are the objects to be clustered and the edges represent the similarity between two objects, based upon the data from that view. When the views of the data are not a network of the objects to be clustered already, the conversion of the data to a view graph can be done by the computationally quick heuristic of using a symmetric k-Nearest Neighbor graph, where $k$ is the square root of the number of vertices, $k=\lfloor\sqrt{n}\rfloor \mathrm{v}$ (Qiao et al. 2018). While this method can be used for creating the graphs, there are many other methods for inferring or learning graphs from data which could easily be used in this methodology (Brugere et al. 2018; Qiao et al. 2018). The end result of the first step is that each view is now a graph where the vertices are the objects to

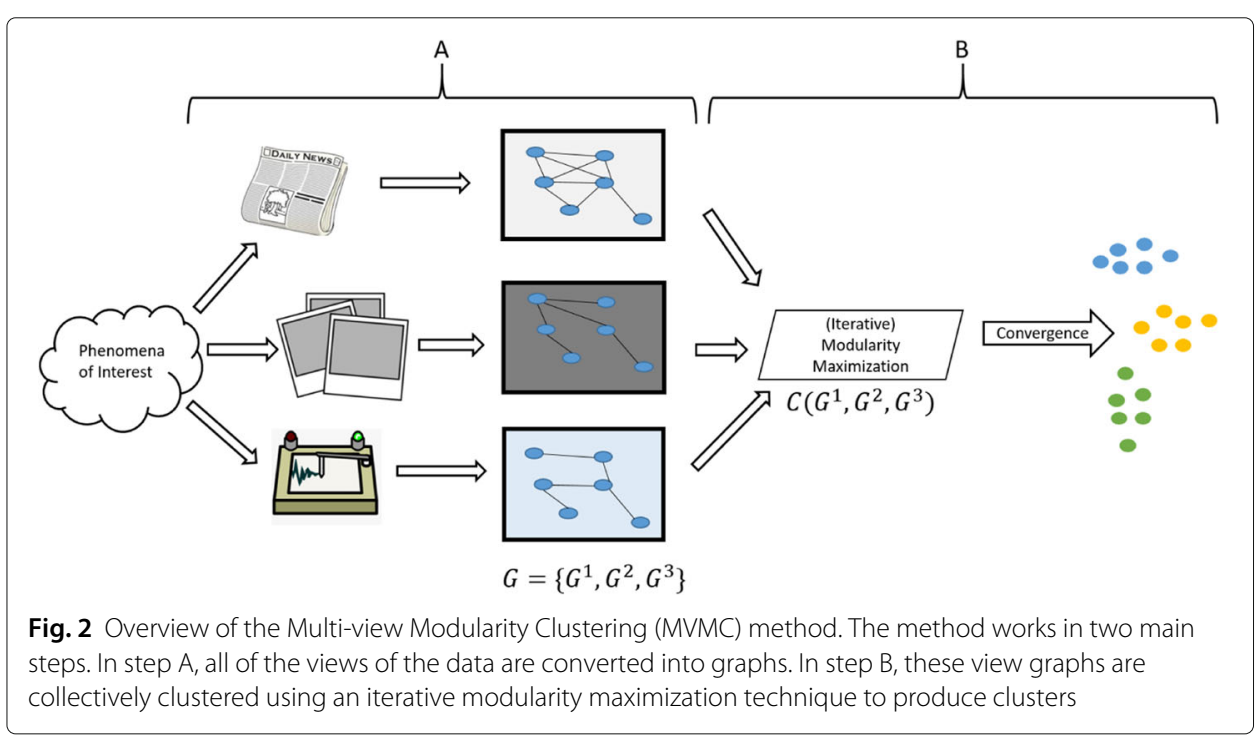


be clustered and the edges represent the propensity to be in the same clusters for that view of the data. That is to say that those vertices connected by edges have more propensity to be in the same clusters than those objects which are not directly connected by edges. Having formed graphs of all of the views, the next step is to use a modularitybased clustering procedure to simultaneously cluster all of the views (B in Fig. 2). This can be done by using a multiplex network modularity maximization procedure (Mucha et al. 2010; Pamfil et al. 2018).

\section{Creation of the view graphs}

The first step of the MVMC procedure is to create graphs of each of the views. For each view, a similarity graph was created. So, for each view graph, an edge represents how similar two objects are with respect to that view. For example, for the text view, an edge indicates that two hashtags share similar text in their tweets, or for the co-occurence view that two hashtags tend to occur with the same set of other hashtags. In order to measure similarity, we first transformed the view data from raw counts (i.e. the number of times a users uses a hashtag) to Term Frequency-Inverse Document Frequency (tf-idf) scores using,

$$
w_{i j}=t f_{i j} \times \frac{n}{d f_{j}}
$$

where $t f_{i j}$ is the number of terms (i.e. users, word tokens, URLs, etc.) occur with hash$\operatorname{tag} i, d f_{j}$ is the number of Hashtags that also feature the $j t h$ term, and $n$ is number of hashtags. This transformation was done in order to down-weight those terms which are common across all hashtags, like 'COVID19' and up-weight those terms which may be significant for cluster structure. While it has been noted in previous works that tfidf can be insufficient for textual information for tweets, the primary reason for this insufficiency that individual tweets have very little text which can accompany them (Vicient and Moreno 2015; Xiao et al. 2014). However, in this methodological set up, many tweets (often hundreds or thousands) are combined for each hashtag, so the text size limitation is not a significant issue. Furthermore, using tf-idf is also very scalable and does not require any kind of a priori semantic knowledge database (Vicient and Moreno 2015). So, for these reason, we have opted to use tf-idf as a means of processing the view data prior to learning the view graphs. Having applied tf-idf to all of the views, the similarity for each of the views was measured by cosine similarity:

$$
s_{i j}=\frac{A_{i} \cdot A_{j}}{\left\|A_{i}\right\| \times\left\|A_{j}\right\|}
$$

Since the data is large in the number of objects that need to be clustered (i.e. hashtags) - on the order of tens of thousands - the graph learning procedure needs to be a computationally efficient one (Qiao et al. 2018). For that reason, we have adopted the heuristic procedure of creating a symmetric k-Nearest Neighbor Graph (k-NN) with the number of nearest neighbors as $k=\sqrt{n}$, where $n$ is the number of objects being clustered Maier et al. (2011, 2009). To symmetrize the k-NN the following step as used: once each object has been connected to $k$ of its nearest neighbors, we have adopted the average strategy, $A^{\prime}=\frac{1}{2} A+A^{T}$, which is common in spectral clustering methods (Qiao et al. 2018; Yu et al. 2017; Zhu et al. 2014). So, at the end of the graph learning step, there is a cosine-similarity weighted, undirected graph for each view of the data. 


\section{Multi-view clustering}

In order to cluster the graphs of all of the views of the data, we use a modularity maximization technique. The function used in the optimization is a modified version of that proposed in (Mucha et al. 2010) for dealing with clustering multi-layer networks. The original multi-layer modularity is given by:

$$
\begin{aligned}
& Q=\sum_{v=1}^{m} \sum_{i j \in E^{v}}\left[A_{i j}^{v}-\frac{\operatorname{deg}(i)^{v} \times \operatorname{deg}(j)^{\nu}}{2 \sum E^{v}}\right] \delta\left(C_{i}^{v}, C_{j}^{v}\right)+\sum_{\nu=1}^{m} \sum_{s \neq v} \sum_{i=1}^{n} \omega_{i}^{s v} \delta\left(C_{i}^{s}, C_{j}^{v}\right) \\
& Q=Q_{\text {intra }}+Q_{\text {inter }}
\end{aligned}
$$

Where $A^{v}$ is the adjacency matrix for a view, $v, \operatorname{deg}(i)^{v}$ is the degree of vertex $i$ in view $v$ (i.e. $\left.\operatorname{deg}(i)^{v}=\sum_{j=1}^{n} A_{i j}^{v}\right), E^{v}$ is the set of edges in the $v$ th graph, $m$ is the number of views, $n$ is the number of vertices, the $\delta$ function returns one if vertices $i$ and $j$ are in the same cluster (i.e. $C_{i}^{v}=C_{j}^{v}$ ) and zero otherwise, $\omega^{s v}$ controls the strength of vertices being in the same cluster between layers, and $C_{i}^{v}$ is the cluster that vertex $i$ belongs to in view $v$. So, this function can be broke into two parts: the first measures the modularity of the clustering within each layer, $Q_{\text {intra }}$ and the second measures the modularity of the clustering between layers, $Q_{\text {inter }}$. For multiplex clustering, or multi-view clustering, where clusters are not allowed to vary between views or layers, this modularity can be reduced to just the sum of the intra-layer modularities. Furthermore, it is also possible to weight the different views, or layers, differently as well. So, a weighted multi-view modularity would have the form of:

$$
Q=\sum_{v=1}^{m} w^{v} \sum_{i j \in E^{\nu}}\left[A_{i j}^{v}-\frac{\operatorname{deg}(i)^{v} \times \operatorname{deg}(j)^{v}}{2 \sum E^{v}}\right] \delta\left(C_{i}, C_{j}\right)
$$

where $w^{v}$ is now the weight of a particular view. Despite its empirical successes and strong grounding in statistical physics, modularity does have an important shortcoming: modularity has a resolution limit. In brief, the resolution limit is when modularity cannot detect clusters present in a network when the network is sufficiently large and communities are sufficiently small (Fortunato and Barthelemy 2007; Lancichinetti and Fortunato 2011). In order to address this shortcoming, several authors have proposed various means of correcting for different resolutions when clustering networks de Santiago and Lamb (2020); Reichardt and Bornholdt (2006); Traag et al. (2011, 2013). One means of addressing the resolution limit is to add a parameter to the modularity to directly account for the resolution that may be present in a network:

$$
Q=\sum_{\nu=1}^{m} w^{\nu} \sum_{i j \in E^{\nu}}\left[A_{i j}^{v}-\gamma^{\nu} \frac{\operatorname{deg}(i)^{\nu} \times \operatorname{deg}(j)^{\nu}}{2 \sum E^{v}}\right] \delta\left(C_{i}, C_{j}\right)
$$

where $\gamma^{v}$ is now a resolution parameter for each view graph that can be set depending on the number of clusters that are present in the network relative to the network's size (Reichardt and Bornholdt 2006). This final equation is now the measure of cluster structure present in the multi-view data, and the objective function for modularity optimization procedures, like Leiden or Louvain.

One issue this final function of multi-view network modularity parameters raises is how to set the view weights and view modularities, without a priori knowing what they should be. Previous works have found what the resolution parameters should be for a network 
given a clustering of that network. In order to set the resolution parameter to an optimal value for a particular graph, the following function is used:

$$
\gamma=\frac{\theta_{\text {in }}-\theta_{\text {out }}}{\log \theta_{\text {in }}-\log \theta_{\text {out }}}
$$

where $\gamma$ is the resolution parameter, and $\theta_{\text {in }}$ and $\theta_{\text {out }}$ are the propensities of having edges internal to clusters or external to clusters respectively. This function was derived by relating modularity maximization to the planted partition Stochastic Block Model (Newman 2016; Pamfil et al. 2018). The intuition behind this function is that when there is a greater propensity to form edges internal rather than external to clusters that the resolution should be higher which would bias the function to find more tightly-knit and possibly smaller communities. Since this formulation relies on knowing the clusters to compute the $\theta$ values, it does not on first glance seem useful for actually clustering a graph. However, the function for computing the resolution has been used in an iterative fashion with modularity-based graph clustering to optimally cluster graphs in reasonably few (i.e. less than 20) iterations (Newman 2016; Pamfil et al. 2018). So, it is possible to iteratively cluster and then update the resolution parameter to both find the optimal clustering of the graph as well as its appropriate resolution parameter.

The same means of computing the optimal resolution parameter can be extended to compute the optimal view weights. Pamfil et al. used the same derivation process of finding the optimal resolution parameters from the relation of Reichardt and Bornholdt modularity to planted partition Stochastic Block Models to obtain the optimal weights for each of the layers in a multiplex graph as:

$$
w_{v}=\frac{\log \theta_{\text {in }}^{v}-\log \theta_{\text {out }}^{v}}{<\log \theta_{\text {in }}^{v}-\log \theta_{\text {out }}^{v}>_{v}}
$$

where $w_{v}$ is the weight given to a view, $v$, and $\theta_{i n}^{v} \theta_{\text {out }}^{v}$ are the propensities for edges to form internal to a cluster or external for the $v$ th view, respectively. $\left\langle._{\nu_{\nu}}\right.$ is the average across all of the views. The intuition behind this derivation is that those views with higher propensity to have edges internal to clusters versus having edges external to clusters relative to the average across all views will have higher weights. So, a view with a better than average propensity to have edges internal to clusters should be weighted more heavily in the modularity calculation for clustering. Once again, as with the resolution parameter, the weight parameter can be determined in an iterative fashion (Pamfil et al. 2018).

Having defined the new objective functions, a new algorithm can then be developed to cluster multi-view data by a modularity maximization optimization. At a high level, the algorithm optimizes the sum of the view-weighted, resolution-corrected modularities in an alternating, iterative scheme. In the first step, the algorithm runs by assigning staring resolution and weight parameters for every view (typically one) and then clustering the graph using a modularity maximization technique like Louvain (Blondel et al. 2008) or Leiden (Traag et al. 2019). These clusters are then fixed and used to compute new resolution and weight parameters. This process is repeated until the resolution and weight parameters no longer change. In the event that the resolution and weight parameters do not converge (which can happen in practice (Pamfil et al. 2018)), the clustering with 
the highest modularity value is chosen as the final clustering. The following psuedocode, Algorithm 1 describes the algorithm in detail ${ }^{2}$.

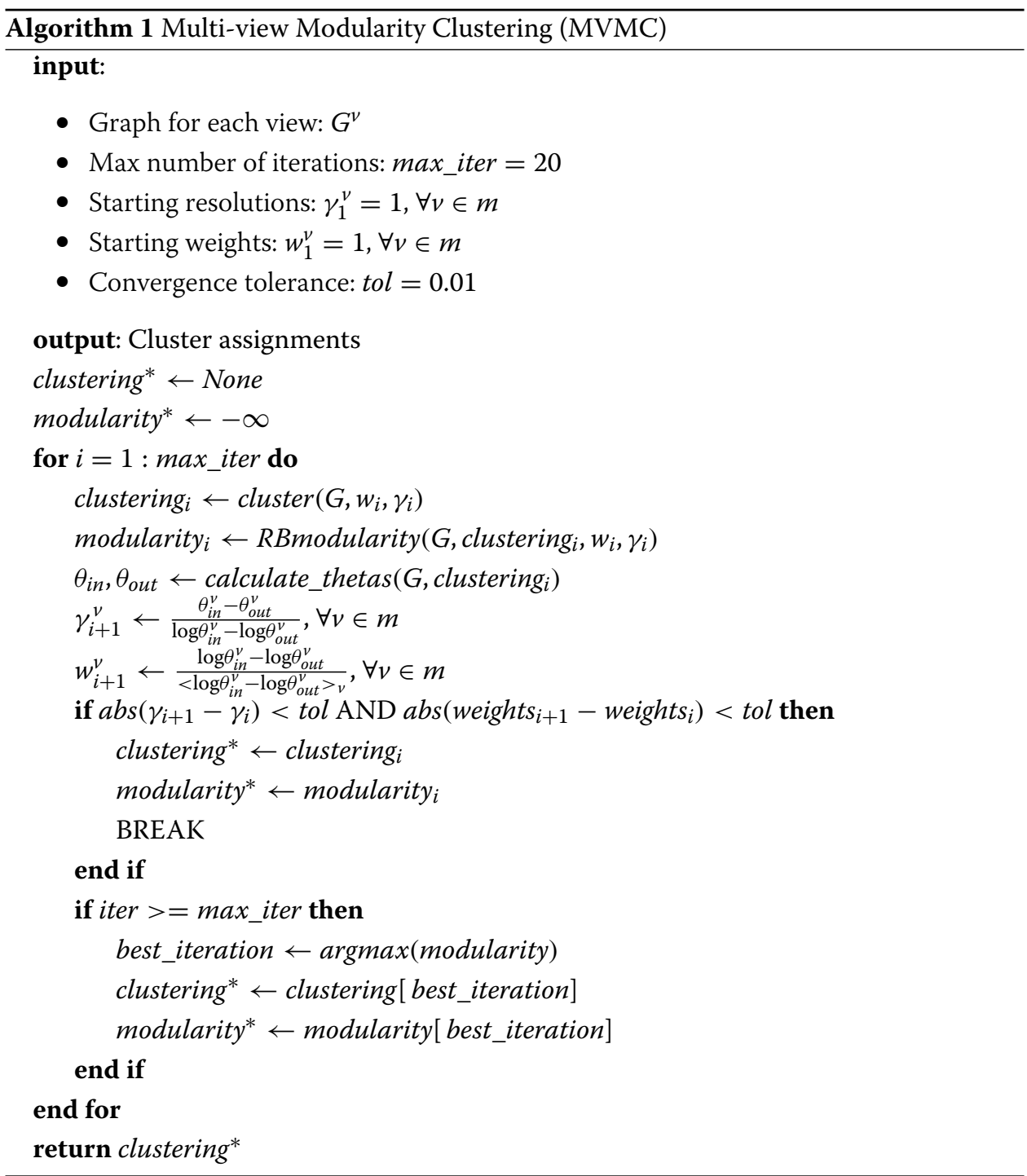

The algorithm begins by initializing all the resolution parameters, $\gamma_{1}^{v}$, and weight parameters, $w_{1}^{v}$ to one (or whatever the user may specify). The algorithm then goes on to cluster the view graphs, $G$, by a modularity maximization technique (i.e. Louvain, Leiden), cluster(), with the current resolution and weight settings. This step of the algorithm can be done with a fast approximate, modularity optimization routine, which typically have computational time complexity of $O(N \log N)$ for each graph (Traag et al. 2019). So this step of the algorithm will have a computational time complexity of $O(m \times N \log N)$. This step of the output of this is then used to determine the propensities for internal edge formation $\theta_{i n}^{v}$, and external edge formation, $\theta_{\text {out }}^{v}$ for each view. These values are then used to update the resolution, $\gamma^{v}$, and weight parameters, $w^{v}$, for each of the views.

${ }^{2}$ Python implementation of the MVMC method is available on the CASOS Institute's GitHub repository at: https:// github.com/CASOS-IDeaS-CMU/Multi-view-Clustering 
If the new weight and resolution parameters are the same as the previous ones (within tolerance), the algorithm then exists and returns the final clustering. If the algorithm fails to converge to stable resolution and weight parameters, within the maximum number of iterations allowed, then the algorithm returns whichever clustering produced the highest modularity. Note, that modularity for this algorithm is the view-weighted, Reichardt and Bornholdt modularity, which incorporates the view resolutions.

One of the important elements in the aforementioned algorithm, Algorithm 1, is the computation of the edge propensities, $\theta$. In order to calculate these edge propensities, we follow the guidance outlined in previous works and assume edges form by a degreecorrected model (Newman 2016; Pamfil et al. 2018). Given a degree corrected model, the expected number of edges that occur internal to clusters is given by:

$$
\begin{aligned}
e_{i n} & =\frac{1}{2} \sum_{c} \sum_{i j \in E} \theta_{i n} \frac{\operatorname{deg}(i) \operatorname{deg}(j)}{2 \sum E} \delta\left(C_{i}, C_{c}\right) \delta\left(C_{j}, C_{c}\right) \\
& =\frac{\theta_{i n}}{4 \sum E} \sum_{c} \kappa_{c}^{2}
\end{aligned}
$$

where $c$ is a cluster and $\kappa_{c}=\sum_{i} \operatorname{deg}(i) \delta\left(C_{i}, C_{c}\right)$, or the sum of the degree of the vertices within cluster $c$. Using the observed number of edges internal to the clusters for the expected number of edges internal to clusters, $e_{i n}$, this equation can then be used to calculate the propensity to form edges internally as:

$$
\theta_{i n}=\frac{e_{i n}}{\sum_{c} \frac{\kappa_{c}^{2}}{4 \sum E}}
$$

Similar to the propensity to form edges internally, the propensity to form edges externally can be derived from the expected external edges under the degree-corrected model as:

$$
\theta_{\text {out }}=\frac{\sum E-e_{\text {in }}}{\sum E-\frac{\sum_{c} \kappa_{c}^{2}}{4 \sum E}}
$$

With these equations, and the assumption of edges forming by a degree-corrected model, the propensities for edges to occur internal or external to a cluster can be calculated. It is important to note, however, that these equations assume the observed edges internal or external to the clusters are equal to the expected edges internal or external to clusters. In practice, these values may actually differ. For example, if every vertex ends up in its own cluster, than no edges will be internal which will lead to the propensity internal term, $\theta_{\text {in }}$ to become zero, and the resolution and weight updates to fail. A similar problem can occur if all the vertices end up in one cluster and so no external edges occur. While at first glance these examples may appear to be edge cases, these problems can also occur in more important cases. For example, if the graph consists of a series of disconnected cliques. The optimal clusters in this situation would then be to put all of the cliques within their own, separate clusters. However, this would result in there being no external edges, and so the resolution and weight updates would fail. In order to address these shortcomings, we have chosen to have a small value substitute for the propensities if there are either no internal edges and/or no external edges. So, while the observed edges can act as a proxy for the expected edges in most cases, the expected edges will always be a nonzero number. With these corrections, the algorithm for computing the propensity values is given in pseudocode by Algorithm 2 . 


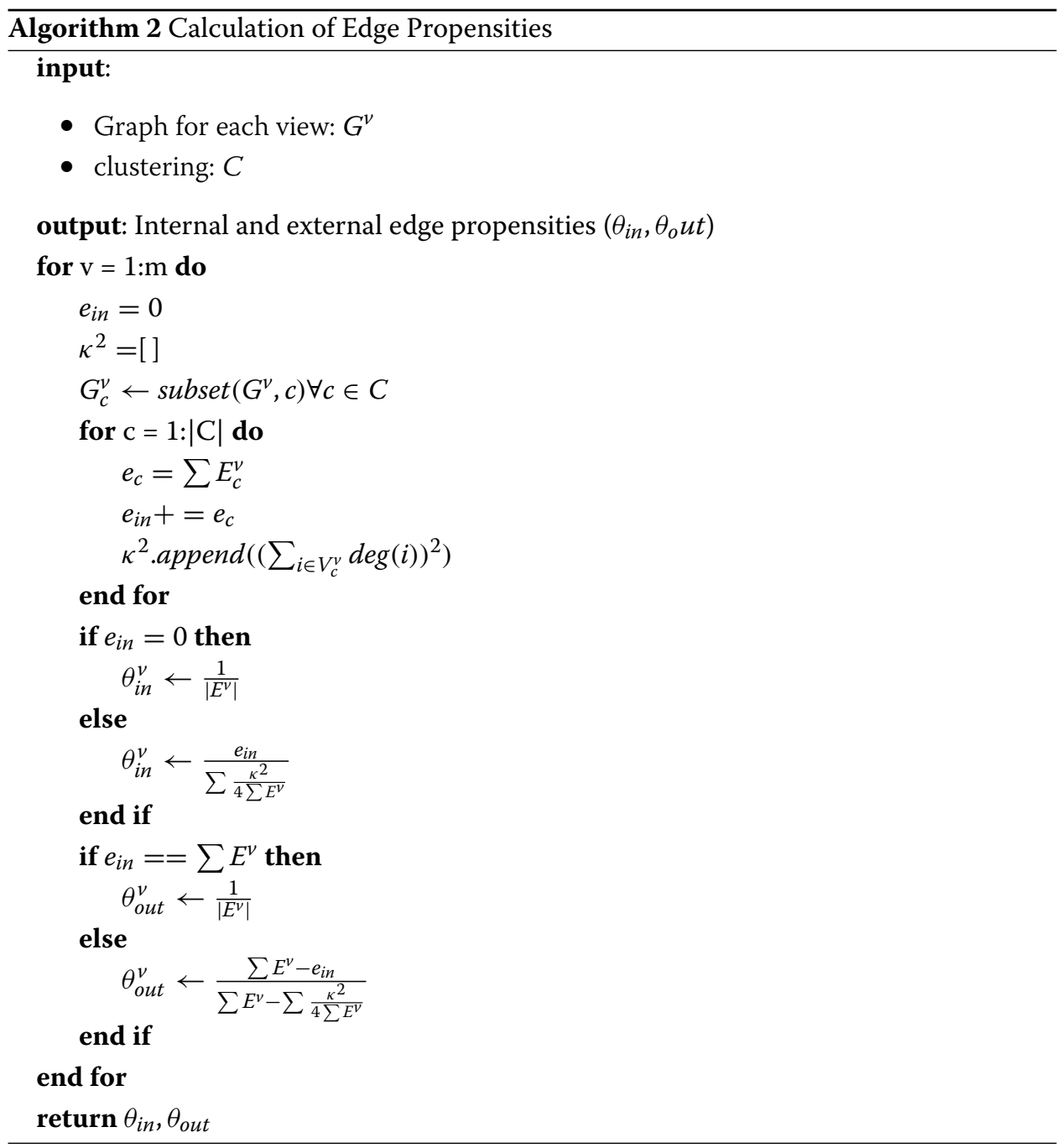

The algorithm goes through each graph to calculate the propensities for each graph separately. For each graph, the algorithm begins by calculating the number of internal edges and the degree-corrected, null-model terms (i.e. $\kappa^{2}$ ) for each of the clusters. Since this step requires going through each vertex in the graph to determine which cluster assignment is internal for that vertex, this step will have $O(N)$ computational time complexity. Then, the algorithm checks as to whether the graph is directed or undirected and whether there are no internal or external edges and then calculates the final propensities for that view graph, $\theta_{i n}^{v}, \theta_{o u t}^{v}$. So, in total the calculation of the edge propensities for all of the graphs will have computational time complexity of $O(N \times m)$, where $m$ is the number of views. Once the propensities have been calculated for all of the view graphs, these are then returned. With the calculation of the edge propensities, $\theta$, we now have everything needed to perform multi-view clustering of the hashtags based upon the four different views of their usage.

\section{Results}

In this section, we describe the multi-view clustering results on the COVID-19 twitter data. In the first section, we provide an overview of the clustering results. In the second 
section, we detail the results of learning graphs on the different views of the data and the insights the graphs give about the data and the cluster structures present within the data. In the third section, we analyze the temporal patterns within the hashtag cluster data. In particular we find three distinct temporal clustering regions. Producing meta-clusters of these temporal clustering regions resulted in some distinct topical clusters, like US politics clusters, a Syrian Civil War cluster, and a online education cluster. In the fourth section we analyze the user bases of the different clusters of hashtags. Finally, in the fifth section we do an in depth analysis of some of the interesting clusters identified within the data.

For clustering the COVID-19 hashtag data, the following parameters for MVMC were used: The initial weights and resolutions were all set to one. The convergence tolerance for the resolutions was set to 0.3 and for the weights to 0.1 . And, the procedure was allowed to run for a maximum of 20 iterations.

\section{Overview of multi-view clustering results}

Multi-view clustering of the COVID-19 twitter data extracted between 20 and 160 clusters of hashtags per day, with a varying size of the clusters. These clusters varied in size and also in number between different days. The following figure, Fig. 3, displays plots of the daily number of clusters and daily cluster size statistics over the full 90-day period.

There is a presence of several small clusters within the daily clusterings. First, on any given day there were between 20 and 80 clusters that had a size of less than five objects. These clusters were almost exclusively composed of either small, locality-specific hashtags, non-English language hashtags, or hashtags that seem to have little relevance to the pandemic. For example, a particular local business, like a car dealership may have a hashtag that is used in a tweet that happens to mention one of the key terms, which was then

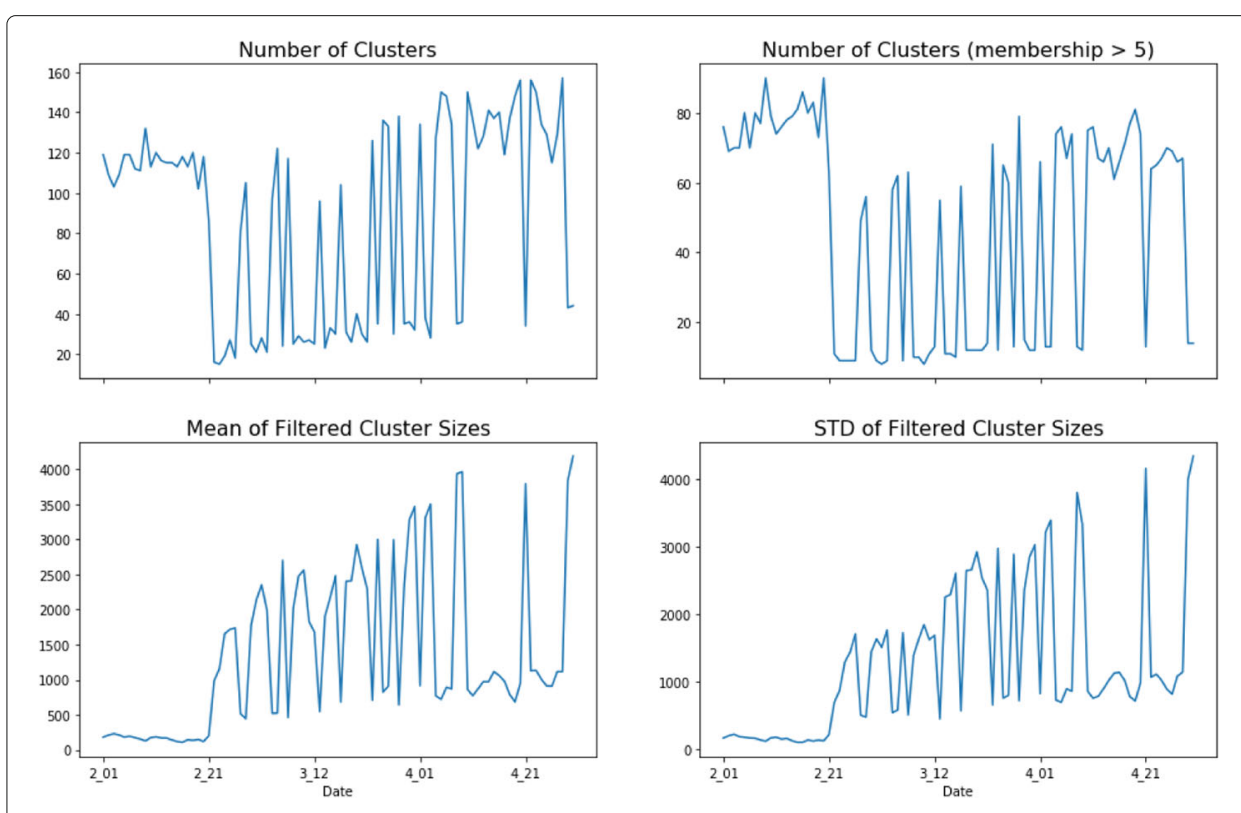

Fig. 3 Daily clustering statistics on clusters produced by the MVMC technique on four views of the hashtags. The daily clusters display three different, temporal patterns of clustering 
retweeted by local residents, which would cause it to be above the initial screening criteria of being used in more than three tweets, but otherwise bear little relation to how any of the other hashtags in the data are used. These small clusters are also often composed of objects that are isolates within one or more of the view graphs, which is what makes them difficult to group into larger clusters. This result illustrates an important point about multi-view clustering of real-world data; the data is often messy and incomplete and requires some degree of additional processing. These arbitrary clusters do not actually contribute much to understanding the data, or the macro-cluster structure of the data, or use of hashtags beyond recognizing that the collection process can produce some noise in clustering results. Removing these small clusters does not affect the overall patterns existing within the clusters, and makes interpretation of the clusters easier.

Second, there are dynamic patterns within the clusters. The clusters start off as many in number and small in size and become fewer in number and larger in size as time passes. Up until the end of February, there are around 80 non-arbitrary clusters with a size of around 250 hashtags. This pattern changes at the end of February where the number of daily hashtag clusters decreases but the size of these clusters increases. This change in the clustering structure over time may indicate that the use of hashtags and their associated discussions begin to congeal into larger discussions over the course of the pandemic. Additionally, there can be large oscillations in numbers of clusters and sizes of clusters between any given set of days. While, there is an increasing trend toward fewer and larger clusters, there are oscillations present within the data, especially during the middle of the time period, around the month of March. While it is not quite clear why these oscillations occur, it was noted in the description of the data that there are weekly periodic patterns within the number of unique hashtags used over time. So, its possible these oscillations are in part due to cyclical, time-dependent patterns in twitter use. This dynamic nature in the clusterings will be further investigated in an upcoming section.

\section{Graph learning results}

In order to better understand the clustering results, we now turn to the analyzing the view graphs. As was described in the "Methodology" section, the graphs for all of the different views were created by a heuristic symmetric k-NN graph learning procedure. This procedure is meant to learn a graph that represents the data. So, graph-theoretic and network science measures can be used to analyze the graphs and thereby better understand the data. The following figures, Fig. 4, display some important graph properties of the different view graphs for each of the daily data sets.

From the graphs, one can first observe that the graph densities follow a pattern that would be expected from the number unique of hashtags. Density initially increases slightly, and then decreases as time moves forward. From the section on the data it is also easy to observe that this pattern is roughly the inverse pattern of the number of daily unique hashtags. So, as expected from observations of real-world networks, as the number of nodes - or unique hashtags, in this case - increases, the density decreases (Newman 2010). Additionally, the text view is consistently the most dense graph while the shared users graph is the least dense graph. Since these graphs were created by a symmetric k-NN graph learning procedure, most of difference in density for the users view is from more groups of overlaps, around the size of $k$, in users between hashtags. When there is more groups of overlaps around the size of $k$ on the features, nearby objects in a k- 


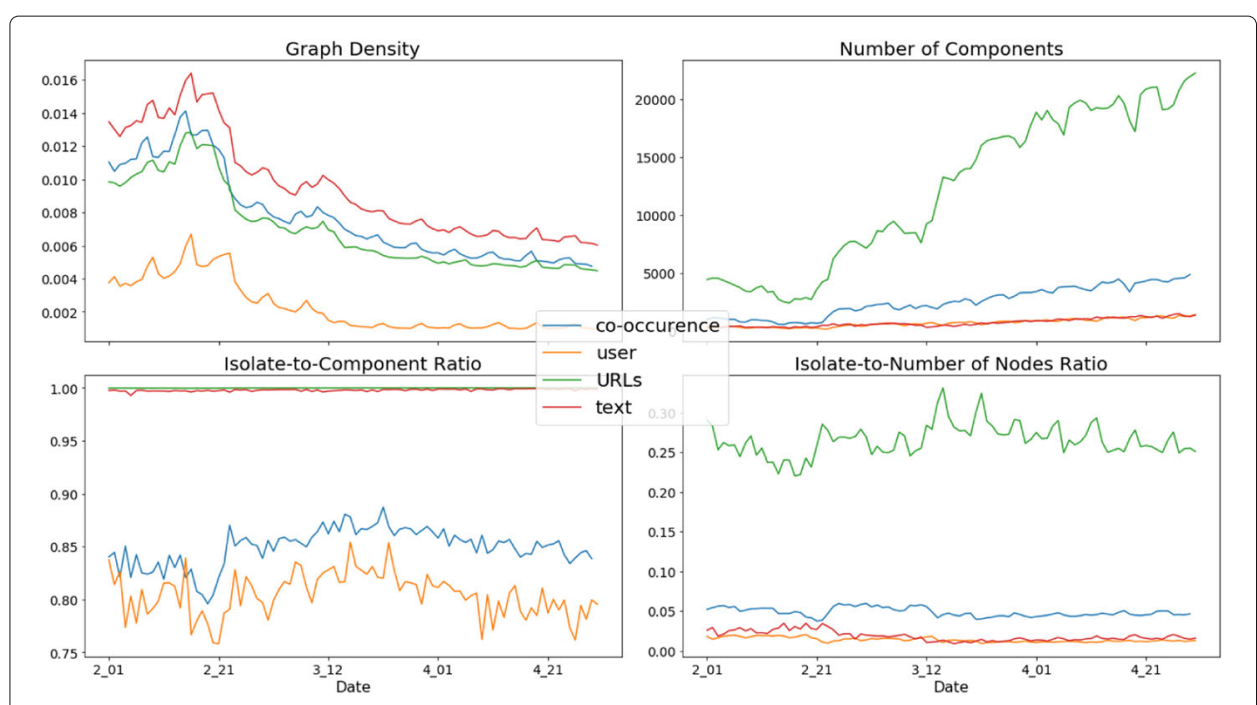

Fig. 4 Graph metrics for each of the daily view graphs. All views but the URL view display useful graph properties for clustering the data

$\mathrm{NN}$ graph tend to be within the $k$ nearest neighbors of each other and that there are only about $k$ neighbors for any given point, which results in fewer edges forming overall once the graph is symmetrized. Conversely, when there are more similar neighbors for each object than the value of $k$, there will be more edges in the symmetrized k-NN graph as two nearby objects, while very similar to each other, may not be within the top- $k$ nearest neighbors of each other.

Second, the component statistics vary considerably between the different views of the data. The URLs view, which measures similarity between hashtags if they co-occur in tweets with the same URLs, has far more components than the other views, and these components - with the exception of one major connected component - are almost always isolates. This is to say that of the hashtags that co-occur with a URL, there are a fair number of URLs that only co-occur with a particular hashtag. This result is also partly an artifact of resolving the Twitter shortened URLs; some of the shortened URLs were unable to resolve to the non-Twitter URL, and Twitter does not always have the same shortened URL for any given URL. Thus, we would expect the URL view to not be particularly useful in finding communities of hashtag use. While much lower on the number of components, a similar pattern is observed with the text mode; those hashtags which are not part of the major connected component are almost always isolates. These hashtags are often rarely used hashtags, typically because they are a common misspelling of a popular hashtag or are a less popular hashtag that occurs with non-English text or no text (i.e. just the hashtag by itself was tweeted). Also, it should be noted that the co-occurrence view, which measures similarity between hashtags based on the other hashtags that those hashtags appear with in a tweet with, has a slightly higher number of components and percentage of isolates than either the user or text views. This is due to the fact that some hashtags never co-occur with another hashtag in a tweet. finally, it is worth noting that for all of the views, the number of components increase with time. These observations suggest that the use of hashtags is becoming more divided into distinct and non-interacting communities. 


\section{Temporal ensemble clustering results}

As has been noted throughout the "Results" and the "Data" sections, the usage of hashtags seems to have some temporal trends and changes over the period of investigation. So, in this section we will analyze the daily clusterings produced by MVMC to understand the temporal nature of the hashtag clusters and hashtag usage. To assess any possible temporal patterns that could exist within the daily clusterings, we analyzed how similar the daily clusterings are to each other. Comparing the similarities between the clusterings can give insight into how stable both the usage of hashtags and the broader discussion topics which use the hashtags are between days. In order to compare the daily clusterings, we opted to use the Adjusted Rand Index (ARI) (Hubert and Arabie 1985). ARI provides a value between zero and one that expresses how similar two clusterings over the same set of objects are. It is frequently used a measure to assess clustering quality in the presence of a ground truth clustering, based on matching permutations of clustering labels. We observed similar similarity measurements for the daily clusterings with other cluster quality measures including Adjusted Mutual Information and Davies-Bouldin Score. In order to measure the ARI between all of the daily clusterings, each clustering has to have the same objects, or hashtags, as every other clustering. So, a set of all of the hashtags used across the entire data set was collected from the filtered hashtag clusters (the filtered hashtag clusters are those clusters which have at least five hashtags in them, for each day). For each daily clustering, if a particular hashtag was not present on that day, it was added to the daily clustering and assigned a dummy cluster label. So, for each day, the clusterings have the same hashtags and those hashtags which do not occur on a particular day are all assigned the same dummy label for that day. Having cross-leveled the hashtags across all of the days in the data set, the pairwise ARI between each day's clustering and every other day's clustering can then be computed. The pairwise ARIs between the daily clusterings are summarized in the following figure, Fig. 5.

From the figure it can be observed that there are some block structures present within the data along with some outlier clusterings. For example, early to mid-February has clusterings which, with the exception of the 13 of February, are all more similar to each other than to any other days' clusterings. This temporal pattern was similarly observed in the clustering overview statistics and MVMC performance statistics. Additionally, clusterings in April also tend to display a block structure whereby the clusterings are more similar to each other than to any other days' clusterings. Outside of these block structures, there are also some outlier clusterings that are not more similar to those clustering which are temporally close. The 13th of February provides an extreme example in that it has very low similarity to every other clustering. Since it appears there are clusters of daily clusterings present within the data along with a pairwise measure of similarity, the clusterings can themselves be clustered. To cluster the daily clusterings, we used the pairwise ARI scores in Agglomerative Hierarchical Clustering with average linkage. It should be noted that clustering a set of clusterings has been used to analyze other temporal streams of data in order to understand dynamic trends within the data (Magelinski and Carley 2019; Masuda and Holme 2019). The following the figure, Fig. 6, displays the full dendrogram for the clustering of the daily clusterings.

From the dendrogram it can be observed that there are indeed clusters of the daily clusterings. And, these clusters tend to consist of temporally nearby clusterings. Thus, it would seem that there are temporal meta-clusters of daily clusterings present in the 


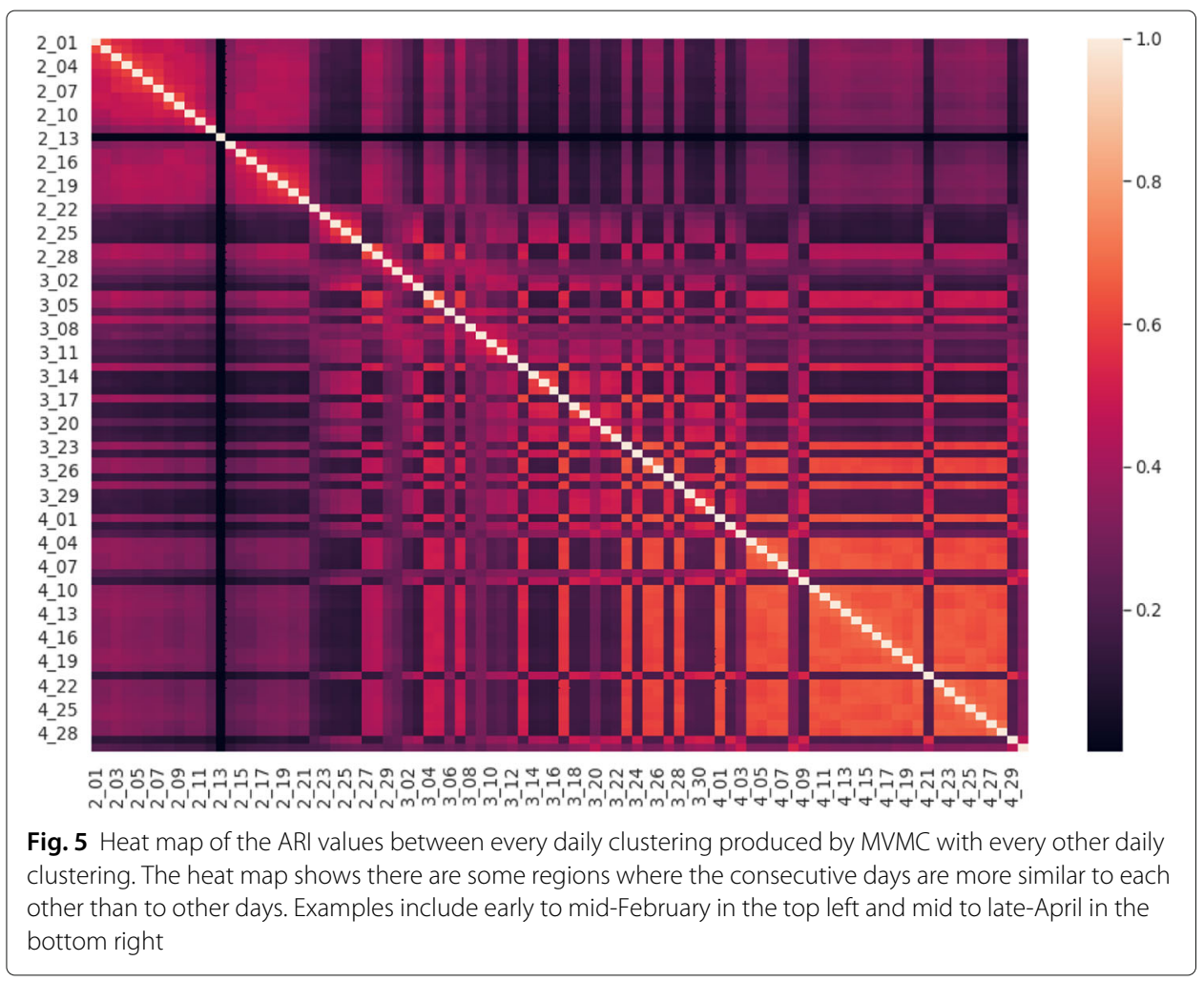

data. To analyze these temporal meta-clusters, we first clustered the clusterings. Based the dendrogram, the clusterings were divided into 5 clusters. Note that the division of the daily clusterings is done without respect to time, but rather is done only on the pairwise ARI between the daily clusterings. Having partitioned the daily clusterings into metaclusters, we can then see if these meta-clusters correspond to any time periods within the

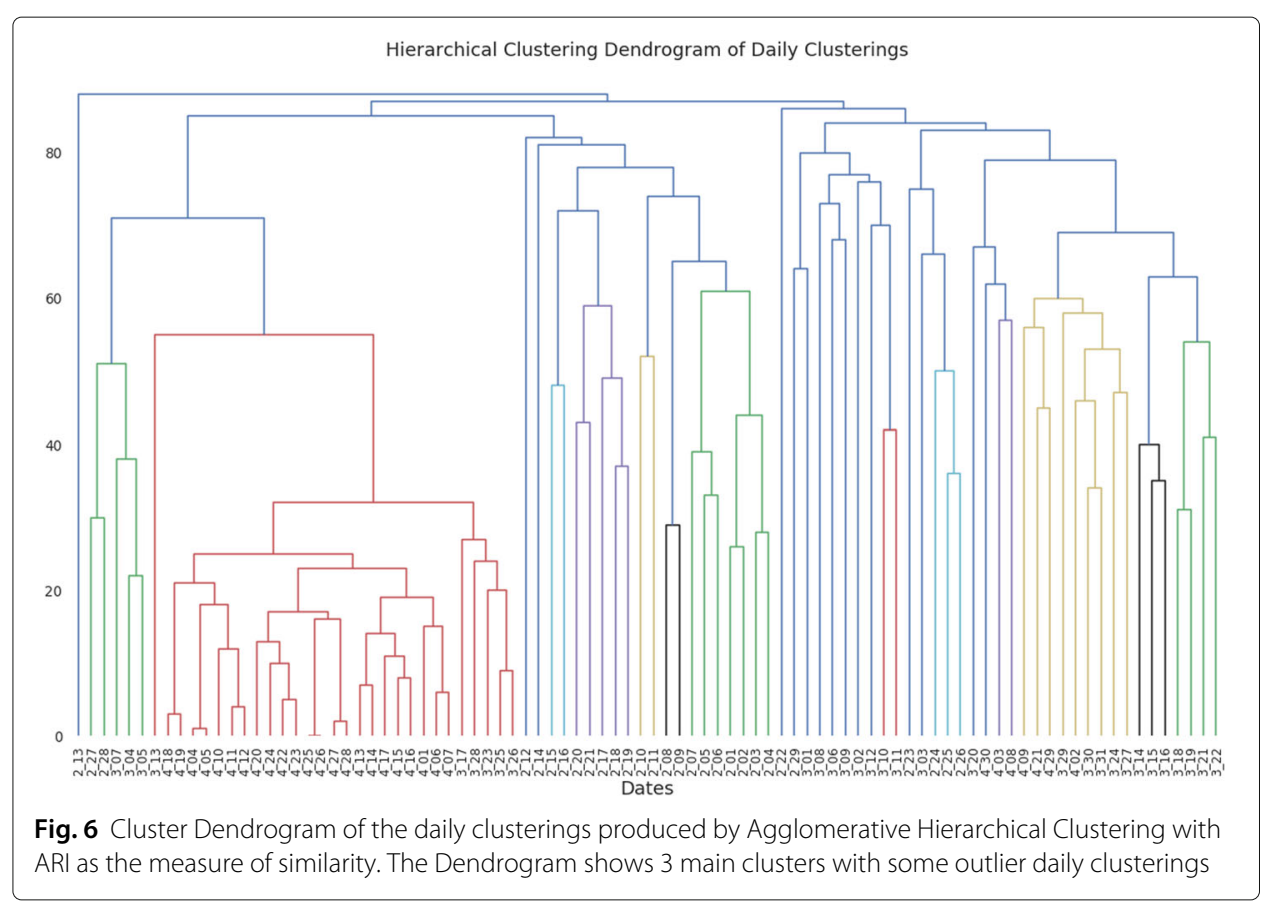


data. The following figure, Fig. 7, displays a plot of the daily clusterings over time versus the meta-cluster that the daily clusterings were partitioned into.

From the figure, there is an observable temporal pattern to the meta-clusters. There is a meta-cluster (label 2) that is exclusively composed of the clusterings from early to lateFebruary. The other two meta-clusters largely contain clusterings from either February 23rd to April 3rd, or April 4th to April 30th. There are also two outlier meta-clusters that consist of only one date, February 13th and February 22nd (labels 3 and 4, respectively). Thus, there is a macro temporal pattern within the daily hashtag clusterings. This temporal pattern in the hashtag clusterings reflects a similar pattern regarding user hashtag ratios that were observed in the "Data" section. In fact the middle period of clusterings, which is the least distinct of the 3 time periods - having meta-cluster 0 and 1 members - corresponds closely to the time period where there were many oscillations in MVMC performance. So, not only does hashtags usage on the user level change over the course of the pandemic, but also the topical groups of hashtags also change over the course of the pandemic. And, it would seem there are two stable periods of hashtag usage that occur during the early stages of the pandemic in February and after the pandemic had been raging globally for some time, in early April.

\section{Analysis of temporally-ensembled clusters}

Having observed three distinct time periods of hashtag clusters, we would now like to get a better sense of how these periods differ in terms of hashtag usage. In order to better understand the hashtag clusters from the different time periods, each of the clusterings making up a meta-clustering are transformed into one clustering through cluster ensembling. This is done for two reasons: First, it makes the selection of which days and which clusters to analyze less arbitrary by reducing the number of clusters that need

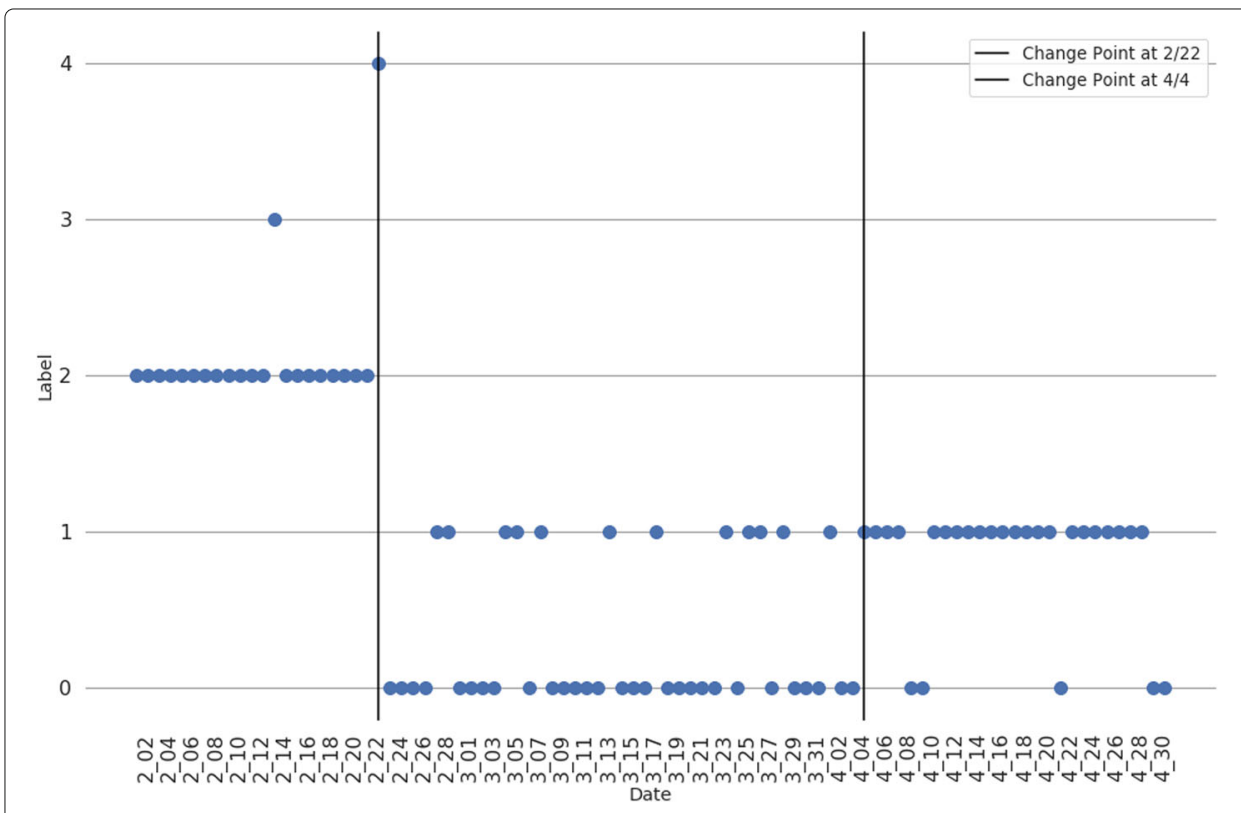

Fig. 7 Plot of daily clustering versus what meta-cluster that daily clustering falls into. Generally, the meta-clusters of the daily clusterings have distinct temporal bounds indicating that daily clusterings have a macro, temporal structure to them 
to be analyzed. Second, producing an ensemble clustering for each of the time periods can better mitigate any daily idiosyncrasies that could affect any given clustering on any given day, and thereby produce a better overall clustering that represents the whole time period. To produce ensemble clusters for each time period the BGPA technique (Fern and Brodley 2004). In contrast to other graph-based methods of cluster ensembling, BGPA treats the ensembling of cluster labels as clustering an object-by-cluster bipartite graph (Boongoen and Iam-On 2018). In addition to showing good empirical performance, the BGPA technique also does not require the construction of any additional graphs, such as an object-to-object shared clusters graphs, which can make the technique more computationally efficient (Cruickshank 2020). So, the BGPA clustering technique was used as it is a very scalable cluster ensembling technique in terms of the number of objects which it can handle and each period has tens of thousands of hashtags (Boongoen and Iam-On 2018). The following table, Table 1, displays a summary of the results for each of the ensembled period meta-clusters.

The ensembled, period meta-clusters produced somewhat different cluster structures for the different time periods. The third period had slightly more clusters and smaller and more regularly sized clusters than the other time periods. This time period also had a higher clustering similarity, in terms of the average pairwise ARI between its constituent daily clusterings. For the first and second time periods, there was often one large cluster that had a size of around 4,400 hashtags while the largest cluster in the third time period was 3,025 hashtags. So, the third time period has a more balanced cluster structure, across the entire time period, than the other two time periods.

To get a better idea of the ensembled clusters, the following table, Table 2 provides a qualitative assessment of the topic of cluster as well as how focused and easily assignable a topic is to each of the clusters in each of the time periods.

Looking at the hashtags present in the clusters of the different ensembled metaclusterings revealed both persistent topical groups and ones which change over time. In every time period, there is always a cluster that has hashtags for breaking news or news sources, a cluster that has business and commerce related hashtags, a cluster that has U.S. Politics-related hashtags, and foreign language clusters - most notably Italian, German, and Spanish. These topical groups indicate that conversations about the global economy, news, and U.S. politics have remained important and consistent topics throughout the pandemic, and that even with English-language collection terms, the discussions occurring around the COVID-19 pandemic are international in nature. In addition to these persistent clusters there are also transient cluster topics that emerge in some time periods but not others. For example, in time period two there is a cluster of hashtags dedicated to online education and a cluster of hashtags concerning online entertainment and entertainment services (i.e. Hulu, Netflix). Both first and third periods contain clusters with

Table 1 Cluster statistics of the ensembled clusterings of the daily clusterings for each time period

\begin{tabular}{lllll}
\hline Period & $\begin{array}{l}\text { Number } \\
\text { of Clusters }\end{array}$ & $\begin{array}{l}\text { Average Size } \\
\text { of Clusters }\end{array}$ & $\begin{array}{l}\text { STD Size } \\
\text { of Clusters }\end{array}$ & $\begin{array}{l}\text { Average Internal } \\
\text { ARI }\end{array}$ \\
\hline February 1 - February 22 & 14 & 751 & 1104 & 0.416 \\
February 23 - April 3 & 13 & 747 & 1093 & 0.348 \\
April 4 - April 30 & 16 & 566 & 691 & 0.536 \\
\hline
\end{tabular}

The ensembled clusterings display similar temporal trends to the other analyses of the data in that attributes like the number of clusters present in each period roughly reflect the numbers of daily clusterings and unique hashtag usage during these periods 
Table 2 Topical labels for the temporally-ensembled meta-clusters

\begin{tabular}{|c|c|c|}
\hline Period 1 & General Topic & Focus Level of Cluster \\
\hline 0 & Multi-language, general use hashtags & low \\
\hline 1 & Multi-lingual coronavirus-specific hashtags & low \\
\hline 2 & News resources related hashtags & medium \\
\hline 3 & Chinese focused hashtags (often of negative sentiment) & high \\
\hline 4 & Thai related hashtags & medium \\
\hline 5 & Economy/Commerce related hashtags & high \\
\hline 6 & U.S. Politics related hashtags & high \\
\hline 7 & technology and business related hashtags & high \\
\hline 8 & Asian-languages hashtags & high \\
\hline 9 & Multi-lingual, anti-racism and health news related hashtag & medium \\
\hline 10 & French language and European related hashtags & medium \\
\hline 11 & Italian language hashtags & high \\
\hline 12 & Arabic script hashtags & high \\
\hline 13 & All-caps hashtags with some Syrian Civil War hashtags & medium \\
\hline Period 2 & General Topic & Focus Level of Cluster \\
\hline 0 & News and U.S. Politics related hashtags & medium \\
\hline 1 & News-resources related hashtags & low \\
\hline 2 & Commerce, Economy, and technology related hashtags & medium \\
\hline 3 & Asian-languages hashtags & medium \\
\hline 4 & Spanish language hashtags & medium \\
\hline 5 & French language hashtags & high \\
\hline 6 & Italian language hashtags & high \\
\hline 7 & Turkish language with some conspiracy theory related hashtags & medium \\
\hline 8 & Online entertianment related hashtags & hmedium \\
\hline 9 & German language hashtags & medium \\
\hline 10 & Arabic script and middle-east related hashtags & high \\
\hline 11 & Australian and British news related hashtags & high \\
\hline 12 & Online education related hashtags & high \\
\hline Period 3 & General Topic & Focus Level of Cluster \\
\hline 0 & Spanish language and many general hashtags & low \\
\hline 1 & Multi-lingual, coronavirus and general hashtags & low \\
\hline 2 & Commerce, Economy, and technology related hashtags & medium \\
\hline 3 & Multi-lingual, coronavirus and general hashtags & low \\
\hline 4 & British news, anti-racism, and medical science related and hashtags & medium \\
\hline 5 & U.S. Politics related hashtags & high \\
\hline 6 & Arabic-script and location related hashtags & medium \\
\hline 7 & Chinese focused hashtags (often of negative sentiment) & high \\
\hline 8 & French language hashtags & high \\
\hline 9 & Italian language hashtags & high \\
\hline 10 & Canadian and climate change related hashtags & high \\
\hline 11 & German language hashtags & medium \\
\hline 12 & Asian languages hashtags & high \\
\hline 13 & Indonesian and surrounding countries related hashtags & medium \\
\hline 14 & Thai language hashtags & high \\
\hline 15 & Turkish language hashtags & high \\
\hline
\end{tabular}

Some clusters had much more focused and readily defined topics than did others. Also, some topics are persistent throughout all three time periods, while some only exist in a time period

negative sentiment hashtags toward the Chinese government and in support of Hong Kong protests. Overall, there are consistent topical clusters of discussion and other topics which emerge and disappear over time. 


\section{User-base analysis of temporally ensembled clusters}

In order to get a better sense of the hashtag clusters found through multi-view clustering and temporal ensembling, we can analyze the users that use the hashtags. In particular, it is of interest to observe whether those individuals which most use a hashtag also frequently use other hashtags from the same cluster. Presence of a small number of users being most active in the use of the hashtags could give insight into whether the topical conversation is being driven by a small group of users or is more of an open, less centrally-dominated topical discussion. To do so, we first found the top third of users for each hashtag in each cluster, across all periods, which we refer to as the 'top users. We then analyzed the number of unique top users for each cluster in each time period. The number of unique top users within any given topical hashtag group can give insight into whether there is a diverse user-base driving the topical discussion or not. The following figure, Fig. 8, shows the number of unique top users for each cluster in each time period.

From the figure it can be observed that there are differences in user bases both between time periods and between clusters. The first and third time periods have generally fewer unique users in each of their clusters than the second time period. This due in a large part to the previous observation that there are more unique users in general on any given day during the second period than there are for the first or third periods. It is worth noting, however, that the total ratio of unique users to total users in both the second and third periods are about the same at 0.202 and 0.206 respectively. That is to say that even as the number of unique users decreases slightly in the third period and that the clusters in the third period do not have as many unique users, the period retains a relatively high number of unique users across the time period. Additionally, there are distinct differences in the number of unique top users between clusters within any of the time periods. This is especially true for the second time period. Generally, this difference in unique users is only partly accounted for by a difference in the size of the clusters as the 0th and 1st clusters are always the largest in any given period but do not have the greatest number of unique users for those time periods. This discrepancy in numbers of unique top users

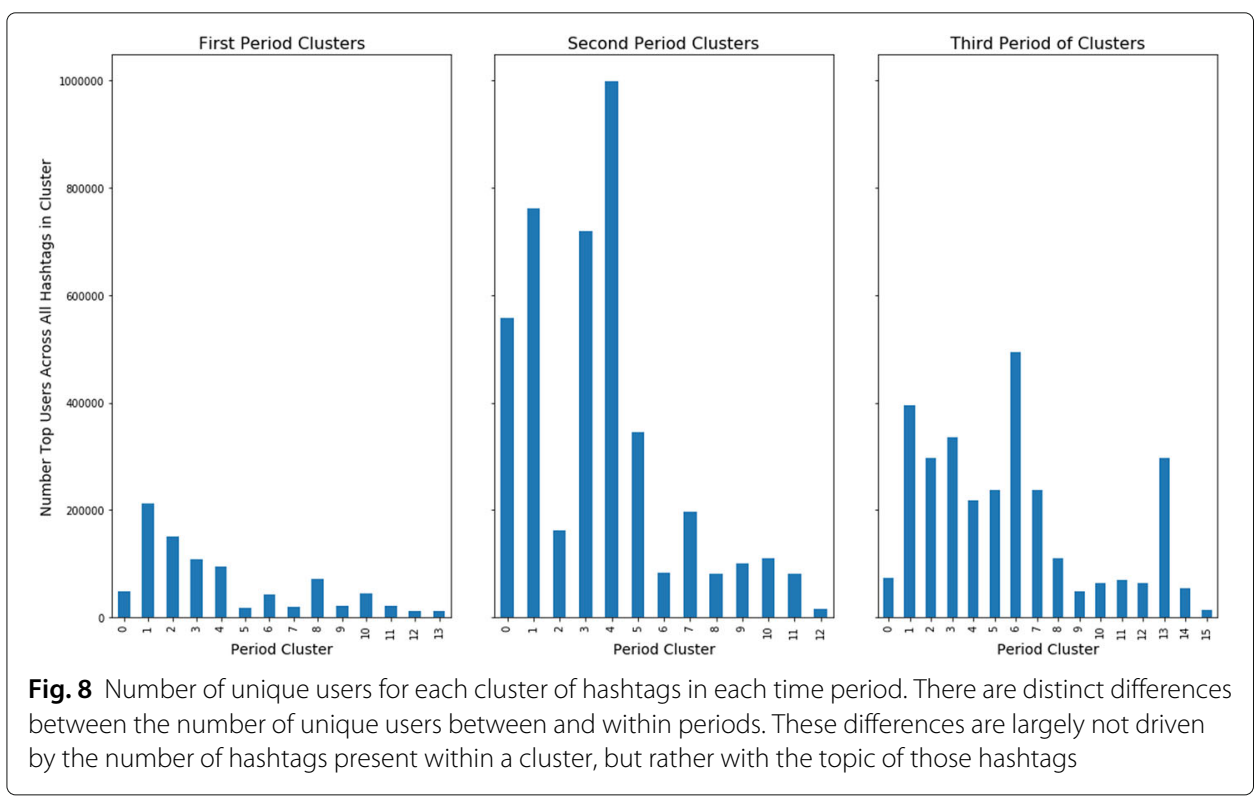


is also a result of the generality of the particular topic of the clusters, with those clusters having more general topics having higher numbers of unique top users. For example, the 4th cluster in the second period contains many hashtags, from many languages, which describe COVID-19, such as '\#COVID-19,' ‘\#covid19', or '\#COVID-19.' So, the topical clusters of hashtags have differences in their top users with some clusters having a very small top user base and others, a larger one.

One of the issues with just looking at the number of unique top users is that hashtags have different numbers of users in general. So, a hashtag could be completely used by a different user in each use, but the hashtag itself is not widely used, which would result in that hashtag having a small unique top user base. This effect extends to clusters where there are clusters of generally less used hashtags. So, I also use a top user score for each cluster which compares the ratio of the number of top unique users for the hashtags versus the number of top unique users if there was no overlap between the top unique users of the hashtags. As a mathematical expression, this top user score for a cluster is given by:

$$
\text { top user score }^{c}=\frac{\sum_{i=1}^{r} \bigcap_{i=1}^{r} \text { top_users }_{i}^{c}}{\sum_{i=1}^{r} \sum \text { top_users }_{i}^{c}}
$$

where $c$ is a particular cluster, $r$ is the number of hashtags present in cluster $c$, and top_users ${ }_{i}^{c}$ is the set of users for hashtag $i$ in cluster $c$. The following figure, Fig. 9, displays the top user scores for each of the clusters in each of the time periods.

From the figure, normalizing the unique top users by the actual hashtag usage across the clusters produces some different results than the previous figures. Some of the smaller clusters with a small user base can actually have a very diverse user base. For example, period one cluster 13, which focuses around the Syrian Civil War, has the highest top user score but a relatively small number of unique users. Another example is period 2 cluster 12, which focuses exclusively on online education-related hashtags, has a small number of unique users but the most diverse user base for the second time period. So, some clusters which can be small in the number of users can have very different

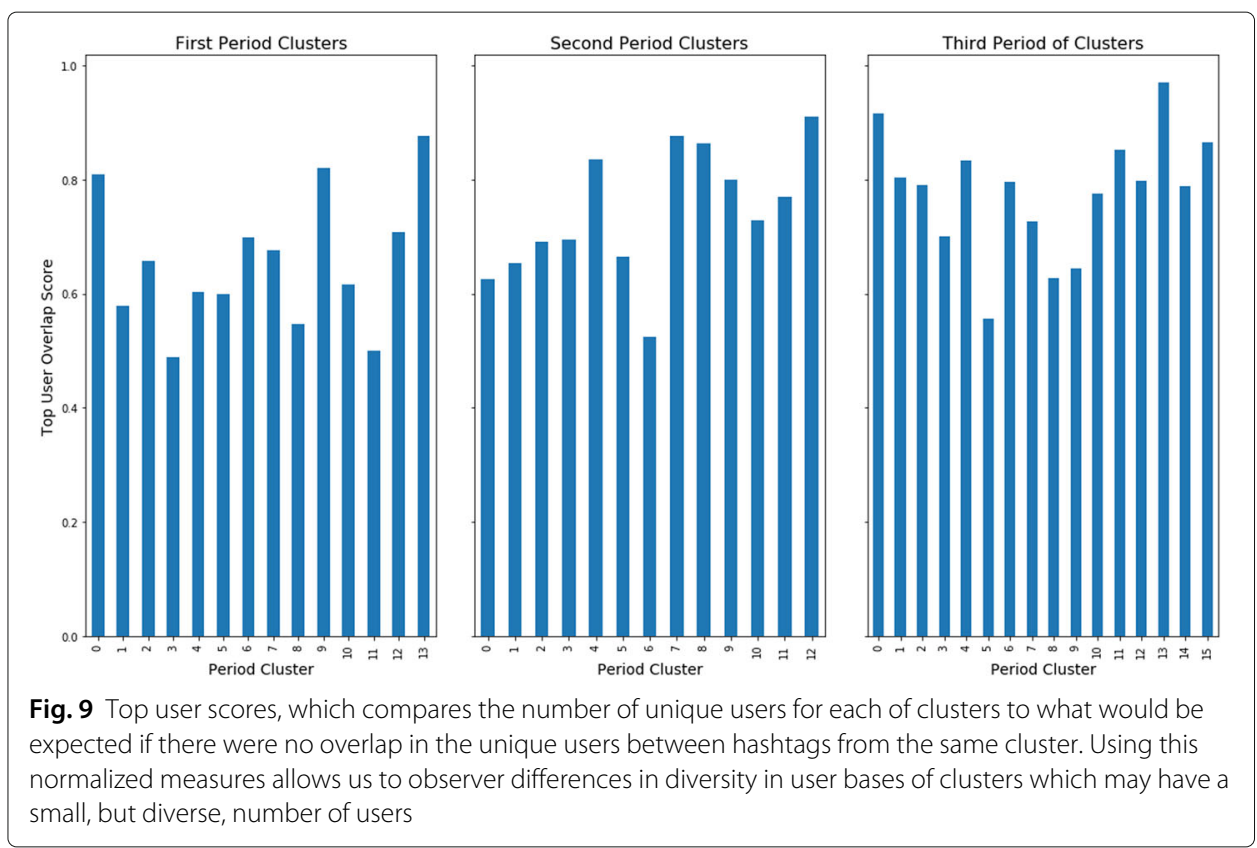


users using the hashtags. This observation would suggest that only analyzing hashtags by creating hashtag-to-hashtag networks based on the shared users is actually insufficient to find clusters of hashtags, which was a similar result observed by other authors (Vicient and Moreno 2015). In essence, two hashtags may be used as part of the same online conversation or topical cluster, but used by completely different users. And so, if one were to link those hashtags by the their shared users, those two hashtags would not be connected in a hashtag-to-hashtag, shared-users network. This disconnect between the two hashtags in the shared users network may result in those hashtags being placed into separate topic groups, when they may be better clustered as being part of the same topic group. Additionally, some of the more mid-sized clusters can have less diverse user bases. For example, period one cluster 3, which focuses on hashtags critical of the Chinese government, has the least diverse user base in the first period, but a fair number of unique users. Period two cluster 6 , which has many Italian-language hashtags, and period 3 cluster 5 , which focuses on U.S. politics, have similar patterns. So, the topic of a cluster tends to drive how diverse the user base of that cluster is, and not the number of hashtags or even the number of unique users.

To further explore the nature of the unique users of hashtags within clusters, we can look at how unique each hashtag's user base is within each cluster. In order to better understand the user base of a particular hashtag we calculated the ratio of the number of unique users that use a hashtag versus the number of times a hashtag is used in a given period. These hashtag user scores can then be combined to analyze each cluster within each of the time periods. The following figure, Fig. 10, displays a box and

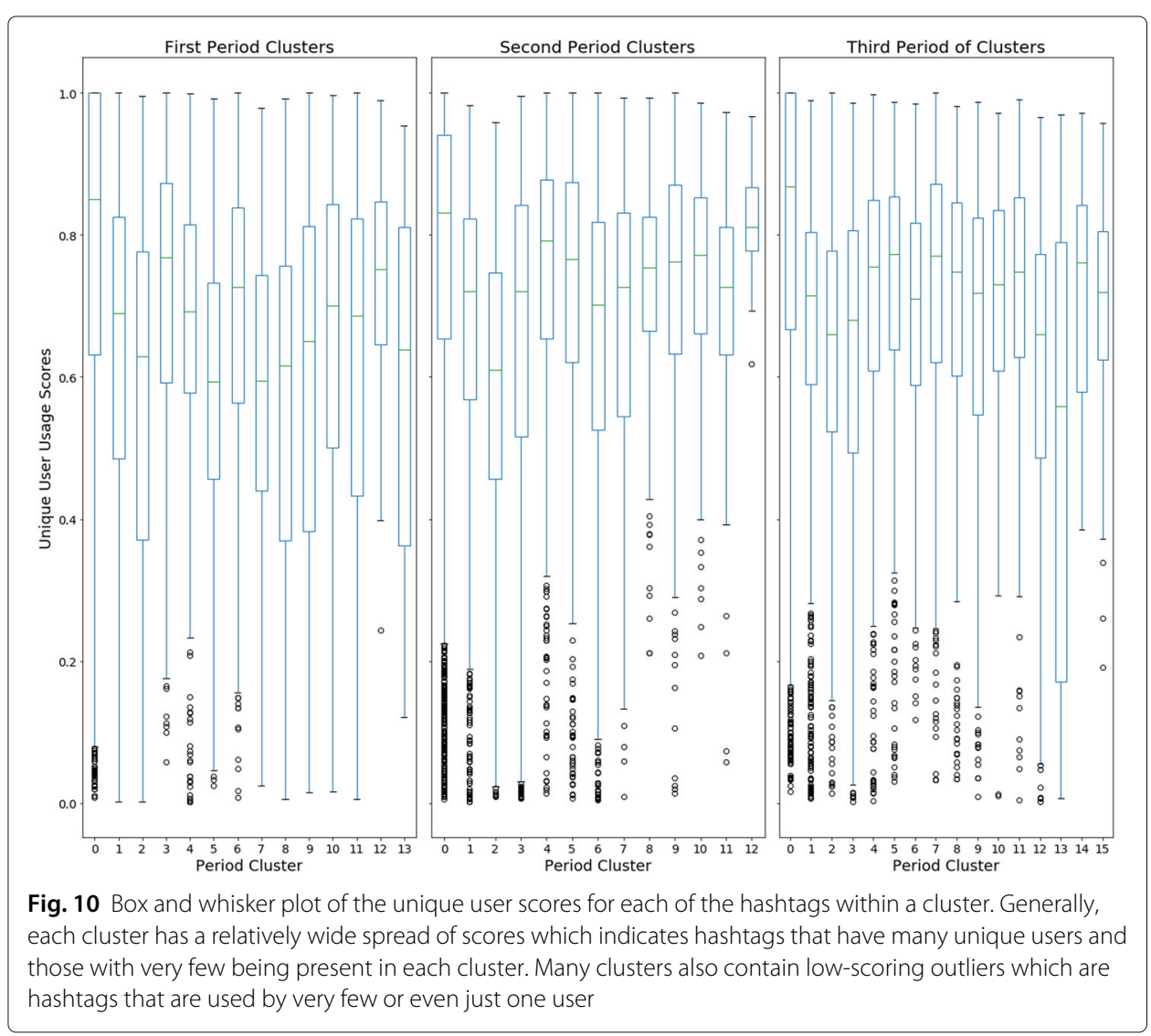


whisker plot of the hashtag user scores for each of the cluster for each of the time periods.

The unique user scores for each of the hashtags within each of the clusters show distinct differences between the clusters within each period. First, it is worth noting that the spread of scores for the different clusters tend to be consistently wide; most clusters have scores ranging from 1.0 to near 0.2 . There are notable exceptions to this, like period 2 cluster 12 which focuses on online education, and period 1 cluster 12 which is an Italianlanguage cluster. Second, while there is a wide range of scores for the hashtags within any given cluster, most clusters often have outlier hashtags with very low user scores. That is to say, that most clusters have at least one hashtag that is often tweeted, but only by a few or one user. So, while the clusters often present distinct themes in their hashtags, the usage of the hashtags within the clusters can vary considerably. Some hashtags have a much more diverse base of usage while some are promulgated by only a few users. This result once again suggests that clusters of hashtags form around topical groups and that all hashtags are not equal, at least in terms of usage, within a topical group.

Overall, the user base of the different hashtag clusters demonstrate that not only are the clusters very different in their user make up, but so to are the user bases for individual hashtags within the clusters. Certain topical areas of hashtag usage, and by extension, discussion, tend to be driven by a small number of users, whereas others have a much more diverse user base. Furthermore, within clusters of hashtags, hashtags can vary quite a lot with their user bases, with some hashtags being promulgated by very few, or even one, users. So, even within a certain topical area of hashtags, a small group of users will control the usage of certain hashtags and possibly parts of the discussion as well.

\section{Detailed analyses of particular clusters}

In this section, we analyze a few of the clusters more deeply. In particular, we analyze the usage statistics of different hashtags within the clusters as well as the verbiage associated with those clusters.

\section{First period, chinese-focused cluster}

The first cluster of interest is a Chinese-focused cluster from the first period. This cluster was composed almost entirely of hashtags relating to either China or Wuhan. It should also be noted that this time period had a hashtag that featured the term 'china' or 'wuhan' in every single cluster except the cluster focused around business and commerce (period 1 , cluster 11) and the cluster focused around U.S. Politics (period 1 cluster 6); around 86\% of the clusters in the first period featured a hashtag with one of these terms. In contrast, $68 \%$ of the clusters in the second period and $50 \%$ of the clusters in the third period had a hashtag with one of these terms. The following table, Table 3, displays a sample of some of the hashtags present within the cluster.

In general, the hashtags within this cluster express negative sentiment towards China and the Chinese government. Some of the most used hashtags within the cluster express support for Hong Kong protests and symbols of frustration with the Chinese government, like '\#LiWenliang' (Corporation 2020). This trend is further emphasized with both those hashtags which are used by a diverse user base (highest original user ratio) and those used by very few users. Overall, there is a mix of anti-Chinese hashtags and pro-Hong Kong and pro-Tibet hashtags present within the cluster. So, in the first period, which are 
Table 3 Top raked hashtags from period 1, cluster 3 which is Chinese-focused in its hashtags

\begin{tabular}{llllll}
\hline $\begin{array}{l}\text { Period 1, cluster 3: } \\
\text { Chinese-Focused }\end{array}$ & Number of & & & & \\
Most Used & Uses & Original User Ratio & Ratio & Lowest User Ratio & Ratio \\
Hashtags & 145252 & WeStandWithHongKong & 1.000 & myedgeprop & 0.058 \\
\hline WuhanVirus & 116401 & HKpolicestate & 1.000 & hongkonggenocide & 0.108 \\
HongKong & 52252 & Catastrophy & 1.000 & usdjpy & 0.112 \\
CCP & 46059 & Darkness & 1.000 & china_is_territorist & 0.122 \\
Hubei & 44336 & ProtestArt & 1.000 & TechJunkieNews & 0.161 \\
Chinese & 31435 & HKexit & 0.998 & EiSamay & 0.165 \\
WuhanPneumonia & 31152 & Hubie & 0.995 & hongkongprotest & 0.176 \\
Coronarivus & 26612 & timelapse & 0.985 & CaptainTripps & 0.196 \\
WuhanCoronavirusOutbreak & 26352 & Stayclam & 0.982 & Análisis & 0.202 \\
LiWenliang & 26324 & PLAAF & 0.982 & EnvironmentHealth & 0.207 \\
HongKongProtests & & Chash & Hashtags with & User \\
\hline
\end{tabular}

Many of the hashtags from this cluster are critical of the Chinese government for either its response to recent protests in Hong Kong or for its response to the COVID-19 outbreak from Wuhan

the early days of the COVID-19 pandemic there is a distinct vein of discussion within the COVID-19 discussions that is expressing negative sentiment toward the Chinese and Chinese government. It is interesting to observe that this negative sentiment is not limited to COVID-19 but also encompasses other issues with the Chinese government to include protests in Hong Kong.

To get a better idea of nature of the discussion that uses these hashtags, we now turn to the text view and the words that co-occur with the hashtags in the cluster. The following figure, Fig. 11 displays a word map of the common words that co-occur with hashtags from the cluster.

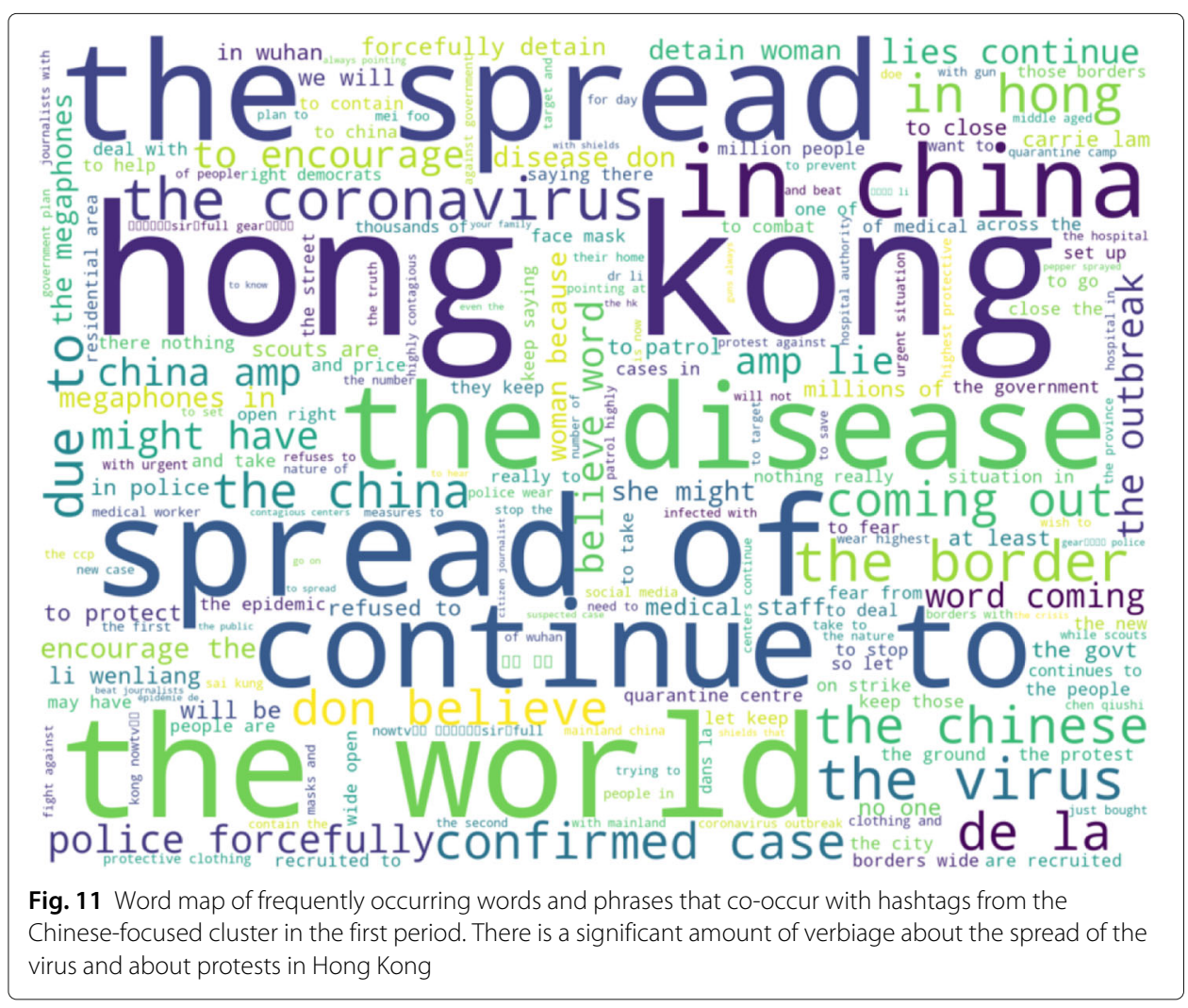


As with the hashtags themselves, the words that often co-occur with the hashtags indicate a focus on the spread of the Coronavirus and Hong Kong related issues. Thus, it would seem that in the early period of the data there is a significant amount of negative discussion surrounding China and the Chinese government both for recent actions in Hong Kong and for being the source of the COVID-19 pandemic. When this result is combined with the user base analysis results of this cluster which indicate that this cluster has the most overlap between the users of its hashtags, it would seem that the discussion and condemnation of Chinese government actions during the early part of the pandemic is driven by relatively few users. This result also indicates that those users who were already critical of the either the Chinese response to Hong Kong protests or to the initial Chinese government reaction to the COVID-19 pandemic may be using the other calamity to draw attention to their calamity of focus. So, while Chinese related terms feature prominently in many of the clusters of hashtags in this period, there is also a distinct cluster of hashtag usage that is critical of the Chinese government.

\section{First period, syrian civil war cluster}

The next cluster of interest is a cluster that features content centered on the Syrian Civil War. At first glance, this is already a strange cluster to have in a data set that was collected based on COVID-19 tweets; there is not an obvious connection between the two entities beside the fact that they are both significant, contemporary calamities. Additionally, the cluster has a high user ratio score, meaning different user accounts are using different hashtags. So, it would seem there is actually a diverse base of users supporting the different hashtags, but the content is almost solely focused on the Syrian Civil War. The following table, Table 4, displays some of the salient hashtags from the cluster.

The hashtags present in this cluster differ from hashtags in other clusters. For one, many of the hashtags in use are all caps versions of other hashtags, such as '\#CORONAVIRUS' for '\#coronavirus'. Second, those hashtags which have very little overlap are almost all of the all caps variety while those with much less user overlap are more widely used and recognized hashtags, like '\#WhiteHelmets'. To get a better idea of the usage of the hashtags present in this cluster, the text co-occurring with the hashtags can be analyzed. The

Table 4 Hashtags from period 1 cluster 13, which has content and some hashtags devoted to the Syrian Civil War

\begin{tabular}{|c|c|c|c|c|c|}
\hline $\begin{array}{l}\text { Period 1, cluster 13: } \\
\text { Syrian Civil War }\end{array}$ & & & & & \\
\hline Most Used & Number of & Hashtag with Highest & User & Hashtags with & User \\
\hline Hashtags & Uses & Original User Ratio & Ratio & Lowest User Ratio & Ratio \\
\hline CORONAVIRUS & 38642 & BILLGATES & 0.955 & AssadGenocide & 0.122 \\
\hline CHINA & 10509 & Nation & 0.944 & Assad_Torture & 0.123 \\
\hline WUHAN & 2397 & ACTUALIZACIÓN & 0.929 & Chemical_Assad & 0.123 \\
\hline NCOV19 & 1543 & LAMORGESE & 0.927 & TheResistance1776 & 0.123 \\
\hline IndianArmy & 1316 & FAKENEWS & 0.908 & AssadCrimes & 0.123 \\
\hline ALERT & 1294 & CINA & 0.899 & PutinAtWar & 0.124 \\
\hline BIOWEAPON & 992 & SINGAPORE & 0.896 & WhiteHelmets & 0.125 \\
\hline Syrie & 981 & BIOWEAPON & 0.860 & InfoWars & 0.215 \\
\hline VIRUS & 910 & BEIJING & 0.859 & TBT & 0.233 \\
\hline AssadGenocide & 831 & ALERT & 0.850 & VIRUSCORONA & 0.241 \\
\hline
\end{tabular}


following figure, Fig. 12, displays a word map for the commonly used text that co-occurs with the hashtags in this cluster.

As with the hashtag themselves, the text co-occuring with the hashtags is different from the other clusters. Phrases like 'link to' and 'to help' feature prominently in the accompanying text and the accompanying text is much more focused in the primary topical area of the cluster than other clusters. Additionally, the text is multi-lingual in that there are mostly English words, but also words from Spanish, French, and others. So, from the text, these hashtags are used to promote awareness of the Syrian Civil War and to draw users to websites to provide support to various organizations involved in the Syrian Civil War. Thus, these hashtags seem to be aimed at using the COVID-19 pandemic to draw user interest to the Syrian Civil War. Additionally, it is also interesting to note that while the text is very uniform in its drawing attention to the Syrian Civil War, the user base is actually very diverse. This cluster has the most diverse user base of any cluster in the first time period. So, these results would suggest that while there is a diverse user base for this cluster, that this diversity is artificial; the users that use the hashtags within this cluster are probably related and possibly being centrally coordinated. At any rate, it is clear from the results that the hashtag usage in this cluster is meant to use the COVID-19 pandemic to draw attention to an entirely different calamity.

\section{Second period, online education cluster}

Another cluster of interest is a cluster of hashtags that only exists in the second time period and focuses exclusively on online education-related hashtags. This cluster contains relatively few hashtags (37 in total) that all relate to online learning. It also exists only in the second period when most of the world entered some form of lock-down to slow the

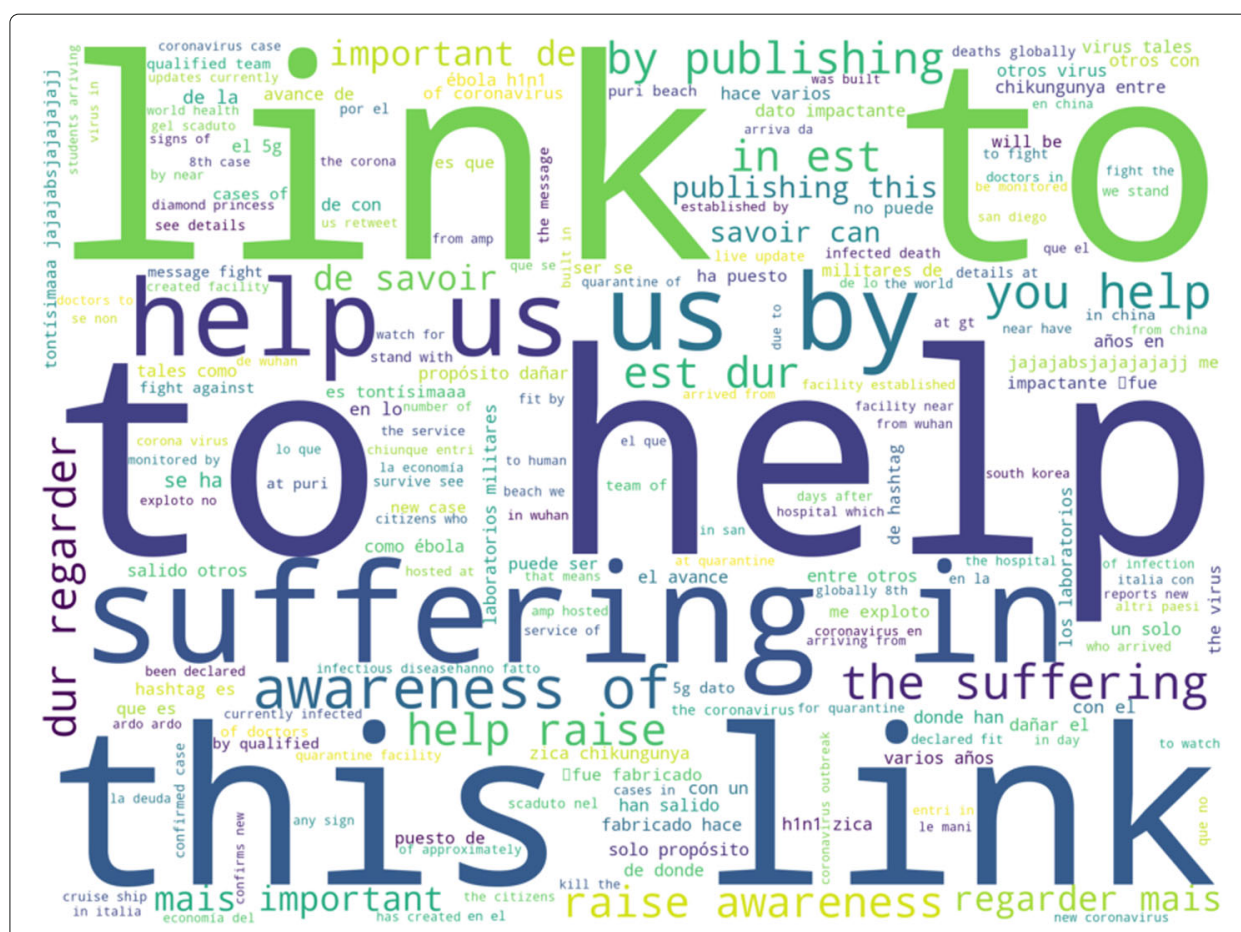

Fig. 12 Word map of commonly occurring phrases and words from period 1, cluster 13. Much of the verbiage seems aimed at directing users to links to support various parties involved in the Syrian Civil War 
Table 5 Hashtags from period two cluster 12 which focus on online education

\begin{tabular}{llllll}
\hline $\begin{array}{l}\text { Period 2, cluster 13: } \\
\text { Online Education } \\
\text { Most Used }\end{array}$ & Number of & Hashtag with Highest & User & Hashtags with & User \\
Hashtags & Uses & Original User Ratio & Ratio & Lowest User Ratio & Ratio \\
\hline education & 9817 & child & 0.967 & Education & 0.619 \\
onlinelearning & 4386 & teaching & 0.907 & Learning & 0.694 \\
edtech & 4673 & AcademicChatter & 0.907 & STEMeducation & 0.712 \\
college & 3530 & college & 0.901 & EdChat & 0.714 \\
AcademicTwitter & 3606 & student & 0.899 & intled & 0.734 \\
distancelearning & 3505 & virtuallearning & 0.886 & highered & 0.739 \\
AcademicChatter & 3204 & universities & 0.879 & edtech & 0.751 \\
edchat & 3594 & AcademicTwitter & 0.876 & HigherEd & 0.761 \\
online & 3235 & students & 0.874 & university & 0.774 \\
STEM & 2936 & distancelearning & 0.867 & education & 0.777 \\
\hline
\end{tabular}

The hashtags used in this cluster have a diverse user base and are focused in that there are no hashtags that are not easily identifiable as being education-related in the cluster

spread of the coronavirus. Despite the few number of hashtags in the cluster, this cluster has the most diverse user base in the second period and has all of its hashtags generally having a diverse usage (the lowest user score for a hashtag in the cluster is 0.619). The following table, Table 5, displays the some of the salient hashtags from the cluster.

As was mentioned previously, all of the hashtags in this cluster relate to education, especially online education, and have diverse user bases. In order to better understand the nature of the usage of the hashtags we turn to the words that co-occur with these hashtags. The following figure, Fig. 13, displays a word map for the frequently used words and phrases from the cluster.

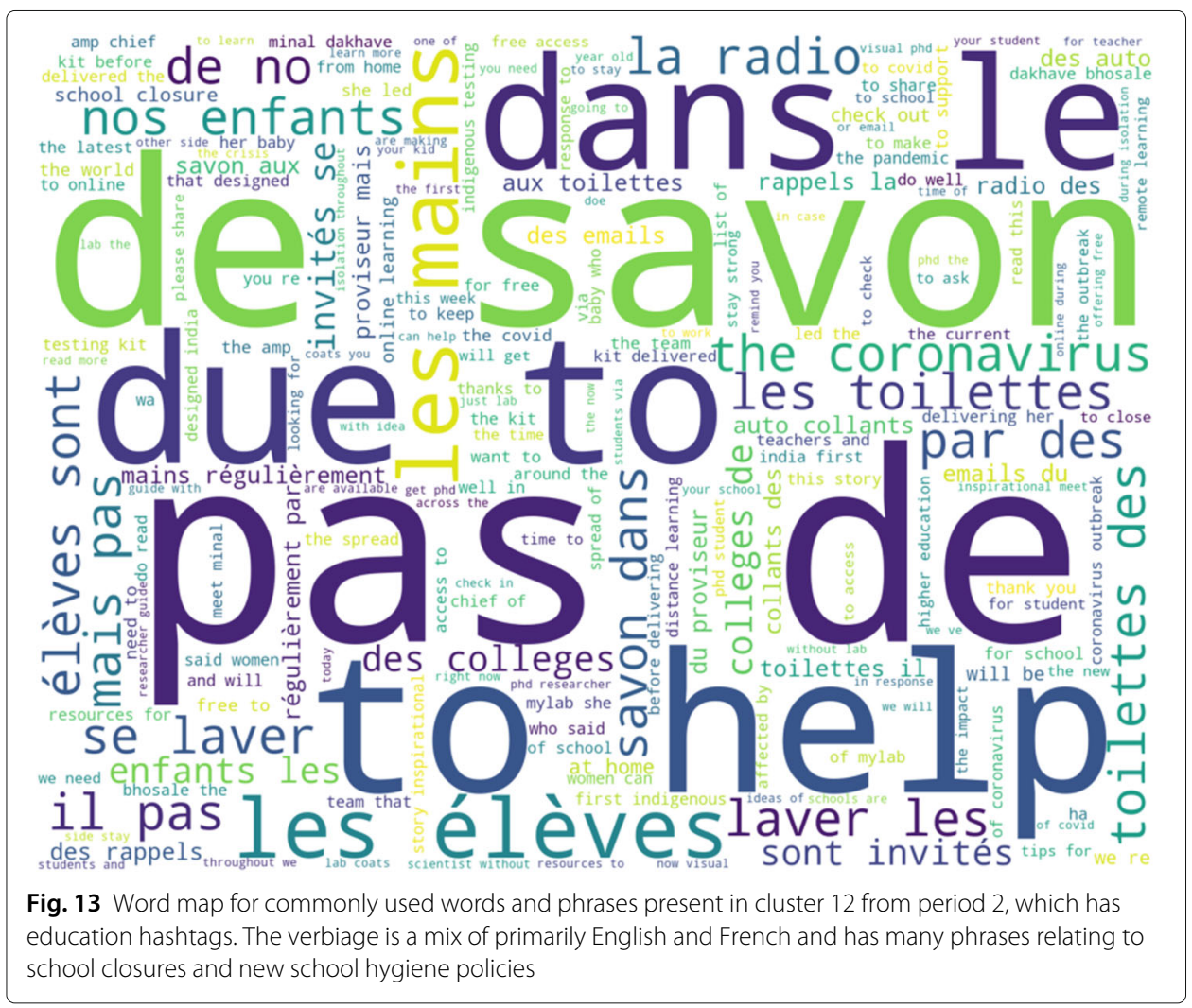


Despite the hashtags all being in English, there is a surprising amount foreign language text, especially French, that co-occurs with these hashtags. Much of the text relates to the closing of schools, and new health-related procedures for schools. These hashtags were used not only to promote online learning solutions and products but also to advertise moving of courses to online and new school-related health procedures. So, this cluster highlights the use of hashtags in order to inform groups of users about both relevant happenings as well as education alternatives during a pandemic. In that sense, this cluster differs from many of the other clusters in that it focuses not only on different content but also seems more aimed at a sharing of non-partisan information to users.

\section{Comparison of U.S. politics focused clusters}

Finally, the U.S. politics focused clusters from periods one and three are analyzed to both understand their content and how the discussion topic of U.S. politics has changed over the course of the COVID-19 pandemic. In the first period, the U.S. politics focused cluster is slightly above average in terms of the user base diversity and has a relatively large number of hashtags at 343. The following table, Table 6 displays the salient hashtags from the cluster.

The hashtags are a mix of political commentary, hashtags related to prominent political figures, and hashtags typically related to conspiracy theories (i.e. '\#QAnon'). Some of the most used hashtags within the cluster relate directly to current U.S. President Donald Trump (i.e. '\#MAGA' and '\#Trump') while some of the most diverse hashtags in the cluster are anti-President Trump (i.e. '\#VoteBlueToEndThisNightmare' and '\#DemCast'). It is also interesting to note that the two hashtags with the least diverse user base - which have scores well outside the inter-quartile range of user scores for the cluster - have only one user who posts both hashtags, 119 and 56 times respectively. So, the cluster contains various hashtags related to various elements of U.S. Politics, including those which are generally associated with partisan content. Thus, in some respects, as with the Syrian War Cluster this cluster contains hashtags which are attempting to use the COVID-19 pandemic in order to draw attention to certain political views or ideas.

Table 6 Hashtags from period one cluster 6 which focuses on U.S. Politics related clusters

\begin{tabular}{llllll}
\hline $\begin{array}{l}\text { Period 1, cluster } \\
\text { 6: U.S. Politics } \\
\text { Most Used }\end{array}$ & Number of & Hashtag with Highest & User & Hashtags with & User \\
Hashtags & Uses & Original User Ratio & Ratio & Lowest User Ratio & Ratio \\
\hline MAGA & 13842 & OpenBorders & 1.000 & hillaryemails & 0.008 \\
Trump & 11976 & Newyork & 0.999 & HAction & 0.018 \\
FakeNews & 9905 & TheGreatAwakeing & 0.994 & StopTheMadness & 0.048 \\
AmericaFirst & 9150 & DemCast & 0.985 & bluelivesmatter & 0.062 \\
QAnon & 8456 & ThesePeopleAreSick & 0.982 & ImpeachTrump & 0.105 \\
GatesFoundation & 7768 & GatesFoundation & 0.981 & TRoom & 0.106 \\
Dobbs & 7752 & IngrahamAngle & 0.980 & ABQ & 0.135 \\
Newyork & 7131 & TrustThePlan & 0.960 & rockoftalk & 0.135 \\
FoxNews & 6209 & VoteBlueToEndThisNightmare & 0.959 & NM & 0.140 \\
FreeZeroHedge & 5736 & JoeBiden & 0.955 & Galaxy & 0.148 \\
\hline $\begin{array}{l}\text { There is a mix of hashtags associated with political news, political personalities and prominent politically-based conspiracy } \\
\text { theories. The hashtags from this cluster also have a wide range of user bases in terms of the uniqueness of the users that use the } \\
\text { hashtags }\end{array}$ & & &
\end{tabular}


To get a better idea of the hashtag usage in the first period's U.S. Politics cluster, I analyzed the text which co-occurs with the hashtags. As with the previous analyses of other clusters, the following figure, Fig. 14, displays the word map for the co-occurring text.

Generally, no phrase or word is particularly dominant in this cluster except for 'the coronavirus' itself. Much of the text mentions the origin of the pandemic in China as well as the global spread of the virus. In this first period U.S. politics cluster, much of the verbiage centers around the origin of the virus and its possible effects. So, much of the discussion from politically-connected hashtags during the first period focuses on China's role in the origin and spread of the COVID-19 pandemic.

Turning to the third period U.S. politics focused cluster, there are similar usage patterns among the hashtags. The following table, Table 7, displays the salient hashtags from the third period U.S. politics cluster.

As with the first period's U.S. politics cluster, many of the hashtags surround political commentary, politically-motivated conspiracy theories, and high profile politicians. Unlike the first period, however, the hashtags with the lowest user scores have larger users bases (i.e. 1 unique user versus 7 for the lowest scoring hashtag). There is also an increase in the number of hashtags being used, 343 in period one versus 610 in period three. To get a better sense of the differences between the two periods, the verbiage of the text cooccurring with the hashtags was then analyzed. The following figure, Fig. 15, displays a word map of the commonly used phrases and words from the cluster

In this time period phrases surrounding personalities become much more used, most especially President Donald Trump. Other personalities like Bill Gates and Joe Biden are also frequently mentioned in the political hashtag tweets. So, from the first period to the

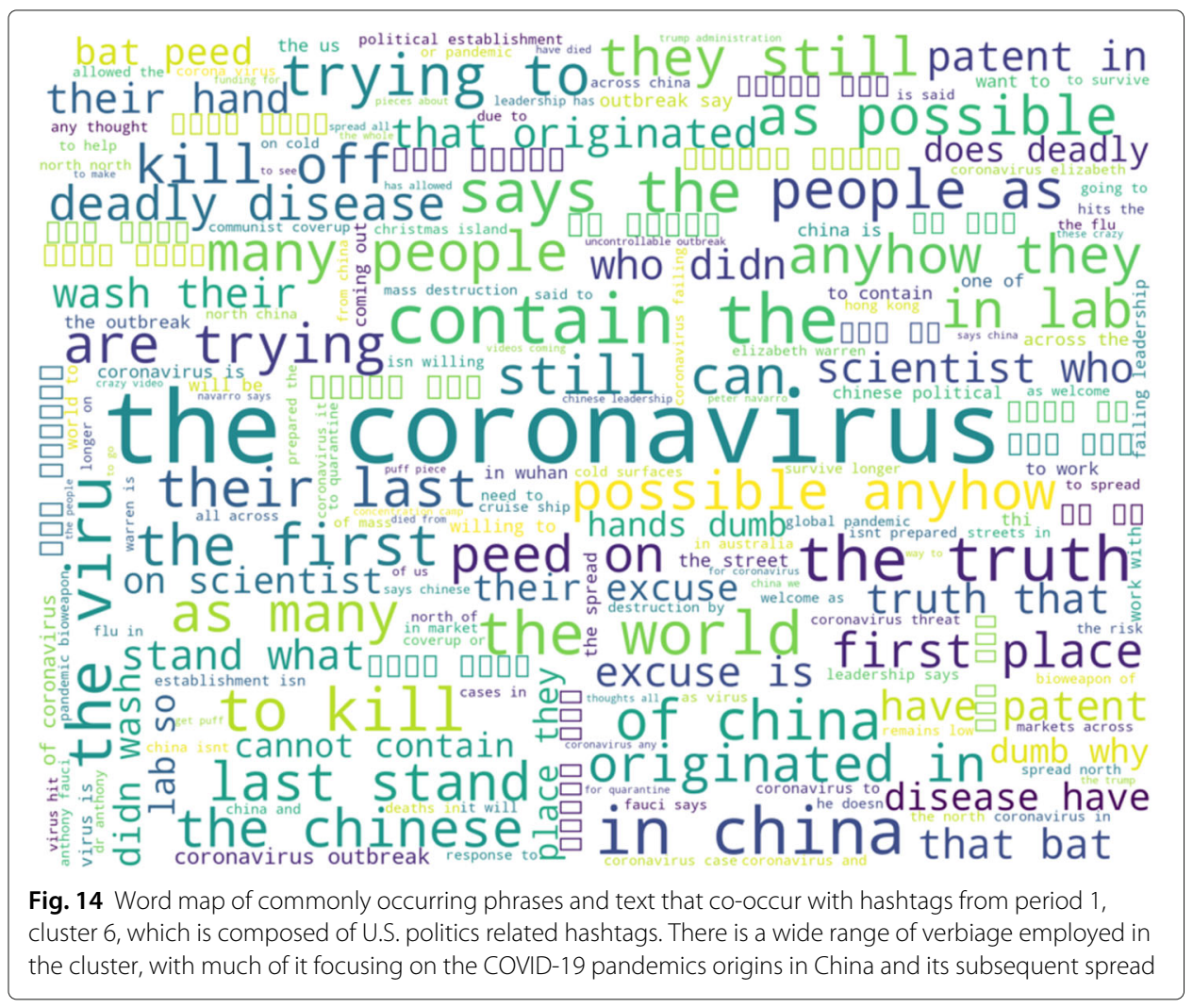


Table 7 Important hashtags from period 3 cluster 5 which has U.S. Politics related hashtags

\begin{tabular}{llllll}
\hline $\begin{array}{l}\text { Period 3, cluster 5: U.S. } \\
\text { Politics } \\
\text { Most Used }\end{array}$ & Number of & & & & \\
Hashtags & Uses & Original User Ratio & Ratio & Lowest User Ratio & Ratio \\
\hline Trump & 189109 & Satanism & 0.988 & hillaryemails & 0.030 \\
FakeNews & 74076 & DeutscheBank & 0.987 & PoliticalViews & 0.037 \\
MAGA & 70999 & Morons & 0.986 & HAction & 0.040 \\
FoxNews & 67531 & Socialists & 0.981 & drudge & 0.051 \\
WWG1WGA & 49348 & ShutltDown & 0.978 & slate & 0.064 \\
KAG & 48339 & AlexJones & 0.977 & newsabq & 0.065 \\
Trump2020 & 46790 & 2ndAmendment & 0.971 & abgfm & 0.067 \\
OneVoice1 & 43457 & hypocrisy & 0.969 & rockoftalk & 0.076 \\
QAnon & 41446 & DrainingTheSwamp & 0.960 & NewsVideo & 0.079 \\
CoronavirusUSA & 34819 & Bullshit & 0.960 & bluelivesmatter & 0.083 \\
\hline Aswith & & & User & Hashtags with & User \\
\hline
\end{tabular}

As with other U.S. politics clusters, this cluster is a mix of hashtags from political news sources, political personalities, and politically-motivated conspiracy theories. Relative to the first period's U.S. Politics cluster, there is an increase in the use of more politically inflammatory hashtags

third period, much of the verbiage shifts from a focus on the virus' origins in China and its spread, to personalities who are often political in nature or who have been linked to the coronavirus, even if erroneously, such as Bill Gates. So, overall there is a shift in the focus of the verbiage paired with the U.S. politics hashtags over the course of the pandemic. This shift generally goes from an external focus to an internal focus relative to the United States.

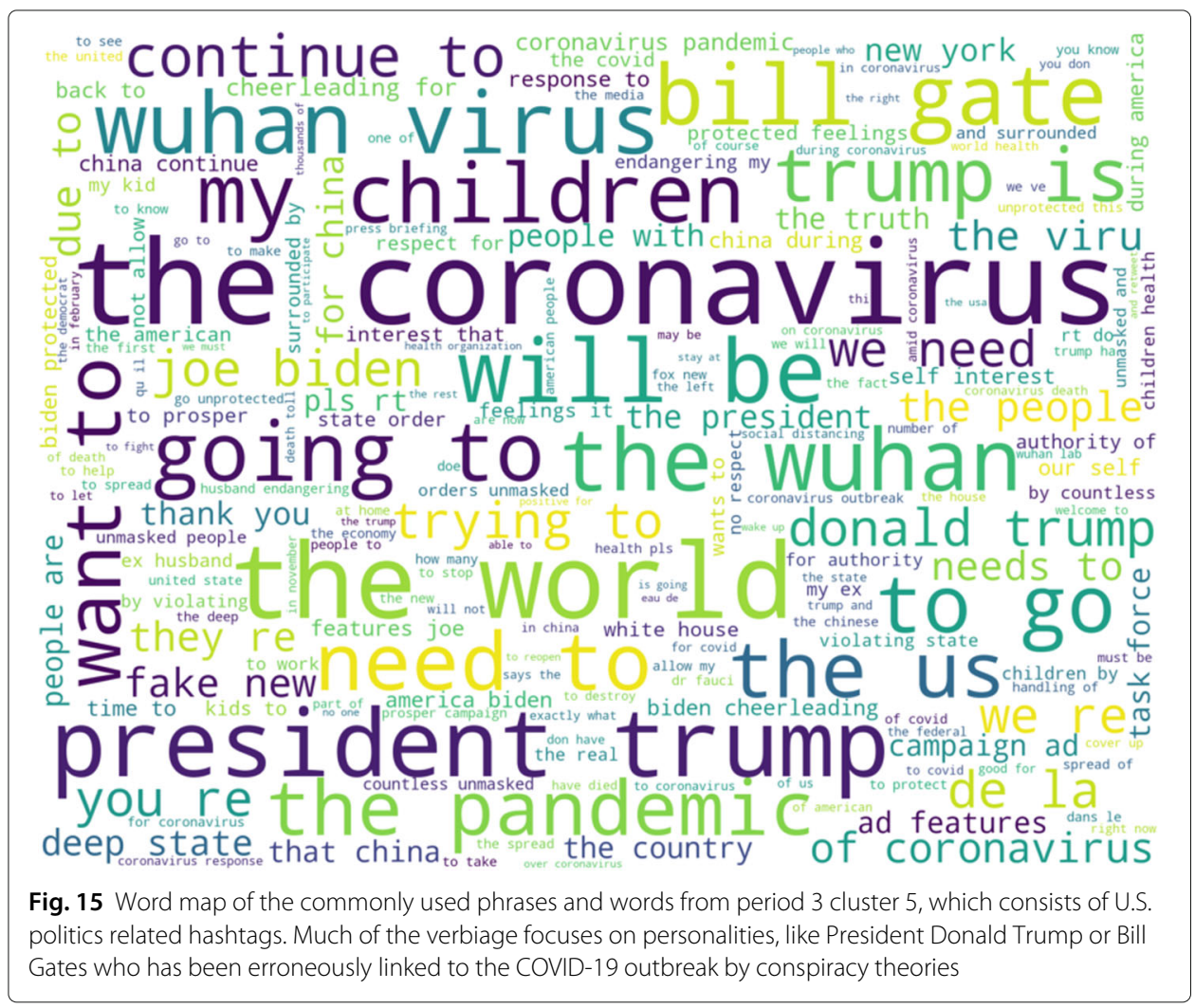


Finally, to get a more complete sense of the differences between the period 1 and period 3 clusters, we directly compare the membership of these two clusters. The user base overlap between the two time periods is small; only $8 \%$ of the union of the two time periods' users are present in both time periods. These users are generally high profile users like politicians or news sources. There is also a $31.8 \%$ overlap in the hashtags used between the two time periods. To get a sense of the differences in membership between these clusters, we analyzed the most important hashtags that exist in only one of the two clusters. It should be noted that many of the hashtags that exist in only one cluster do exist in the other time period that their cluster does not belong to, but not within the U.S. politics cluster of that time period. The following table, Table 8 , displays a side-by-side comparison of the hashtags between the two clusters.

There are some distinct differences in those hashtags only used in one of the clusters and not in the other. First, the salient period one only hashtags feature Australian-related hashtags like '\#coronavirusaustralia' and '\#lnpfail' which are not in period three. Also, there is a rise in the use of conspiracy-related hashtags in the third period only hashtags, such as '\#5g,' ‘deepstate, '\#antivaxx' and more inflammatory hashtags like '\#morons' or '\#senatorforsale.' So, there is not only a regional shift in terms of the difference in the U.S. politics hashtags between periods one and three, but also one toward more polarizing and contentious hashtags over time as well.

Overall, the cluster of U.S. politics-related hashtags differs over the course of the pandemic. The use of some hashtags (approximately a third) remains the same, but the verbiage associated with those hashtags changes from a spread of the disease and Chinese focus to a personality and conspiracy-theory focus. Additionally, the hashtags used in only one time period also show some distinct differences between the time periods. So, while an easily defined topical discussion characterized by the hashtags being used can be persistent over the course of the pandemic, the nature of that topical discussion cluster changes. It is also worth noting that known conspiracy theory related hashtags are always present in the U.S. politics cluster, which demonstrates an strong connection between the two over the course of the COVID-19 pandemic.

\section{Discussion}

There are several findings from this study and results to inspire future research. First, through a scalable technique, like MVMC, it is possible to extend multi-view clustering to a task like clustering hashtags in large-scale social media data. Large scale social media data often requires clustering of tens or even hundreds of thousands of objects and the ability to handle partially incomplete data. The MVMC procedure can successfully deal with both of these conditions in the data and produce meaningful clusters. Using a python implementation of the procedure on a Ubuntu Linux server with just one core active could cluster any given day's data, which would typically require clustering tens of thousands of objects, in a few hours. Use of the MVMC technique also found that certain views, in their current form of feature representation, like URLs which co-occur with hashtags in tweets, were not useful in finding a cluster structure in the hashtags. Also the use of multiview clustering on hashtag can ameliorate problems with previous attempts to cluster hashtags based on just one view. For example, incorporating text and shared users can overcome the observed phenomenon where two hashtags are very related in usage, and should be clustered, but are never used by the same users. It should be noted though, 


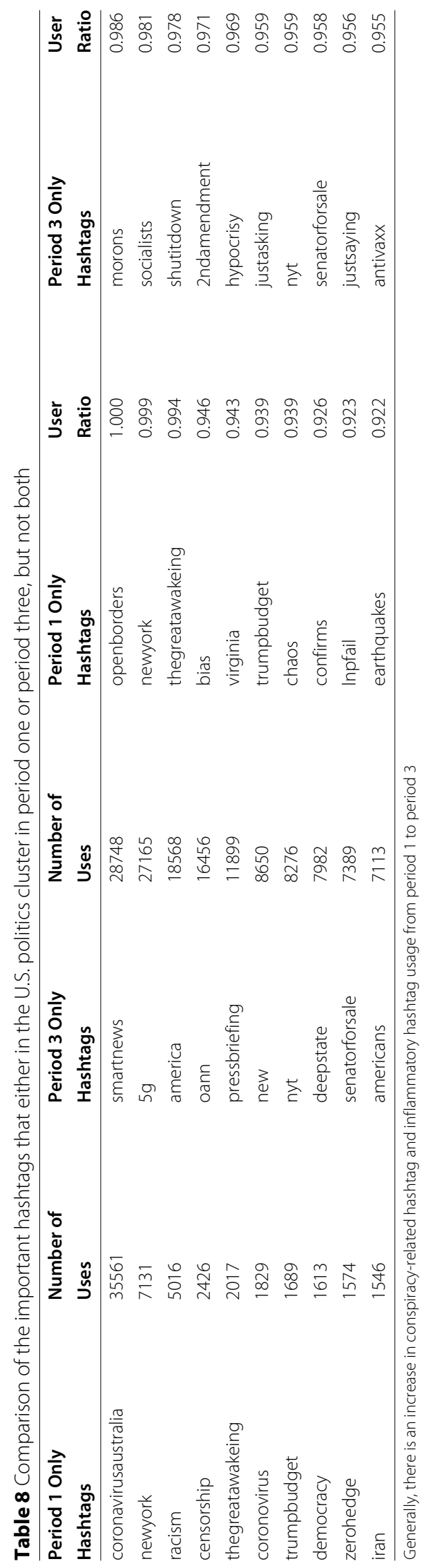


that even though their is robustness to the lack of data of in a particular view, selection of the appropriate views remains a challenge for the method. Thus, through a technique like MVMC it is possible to incorporate all of the previous research on clustering hashtags, that have used co-occurring text or users, into one cohesive model and clustering.

Overall, from the experiments conducted in this study, the MVMC technique is scalable to large amounts of data (on the order of tens of thousands or hundreds of thousands of objects) and to incomplete views of the data. As has mentioned previously, most multiview clustering techniques have not been empirically tested for their performance on large scale data sets. Since many of the successful techniques from multi-view clustering require matrix or tensor factorizations, which can be computationally expensive (i.e. $O\left(N^{3}\right)$ ), it would be expected that they may not scale well to large data sets. In contrast to this, the MVMC procedure has been shown, by the experiments in this study, of being capable of being used on large data sets. Additionally, the MVMC technique can overcome some degree of partially incomplete data. One of the benefits of the multiview clustering paradigm is its ability to utilize more than view of the data, which can provide complementary information across the views of the data sets to address possible missing information in the views. In this work, we empirically observed that the MVMC leverages this benefit of a multi-view clustering to be able to better cluster objects in the presence of those objects having either missing or intentionally obfuscated relationships in some of the views of the data. So, the MVMC procedure, and multi-view clustering more generally, present a means of clustering social-based data that is more robust to things like partially complete views of the data and is capable of scaling to large volumes of data.

Second, hashtag usage patterns during the COVID-19 pandemic displayed dynamic behavior at both the individual and cluster levels. While the data collected is certainly an incomplete picture of the discussions happening on twitter due to API restrictions and the terms used to create the data, it can still offer some insights. From the early days of public awareness about the pandemic in February of 2020, there was an increase in the number of unique hashtags being used and the number of unique users participating in the COVID-19 discussion on twitter. The usage patterns of hashtags, however, varied over the course of the pandemic with there being an initially high rate of hashtag usage that drops when the number of unique users increase, and then increases again as the number of unique users levels of in late March/ early April. This suggests that when a major exogenous shock happens to social media users, like a pandemic, there will be an initial phase of interaction without hashtags, and then a move to start re-using hashtags, likely as a tool to aid in finding and participating in discussions.

At the cluster level, the data showed there were three main periods of clusters of hashtags present in the data. The daily hashtag clusterings could themselves be clustered into three distinct time periods of clusterings based solely on the pairwise similarity between the daily clusterings. In general, the cluster structure of hashtags went from a large number of small clusters to fewer, larger clusters and then back to smaller more numerous clusters. This macro temporal pattern in the cluster structure mirrors those findings from the use of individual hashtags and supports the conclusions that there was a surge in COVID-19 twitter discussion which produced an intermediary period of hashtag usage which then settled into a new pattern of hashtag usage different from what was observed prior to the user surge. 
Using the knowledge that the daily hashtag clustering breaks into three periods, we then created an ensemble clustering for each of these periods. This ensemble clustering allows for a clustering analysis of a prototypical clustering for the entire time period. The results of the analyses of these ensembled clusterings produced some interesting insights into the nature of some of the topical discussions happening during the pandemic. Firstly, there are some topics which have been persistent over the course of the pandemic, like commerce, the economy, U.S. politics, and news. Other topical groups like online education or negative-sentiment discussion about the Chinese government are more transitory over the course of the epidemic. From these clusters it was also observed that some topical groups are intending to direct COVID-19 discussion to other topics like the Syrian Civil War or protests in Hong Kong. So, it would seem from the nature of the clusters present that there is the presence and use of hashtags that are meant to use the COVID19 pandemic to draw attention to other causes or ideas. So, hashtags can not only be a means helping users to find and participate in discussions but also as means of shaping user engagement and the discussions themselves.

From the results presented in this work there are several avenues for future research. First, this study focused on the use of hashtags in order to understand topical discussions taking place, which naturally discounts users who do not use hashtags. As was seen in the "Data" section, there is a sizeable amount of the population of users that are posting content related to the COVID-19 discussions that do not use hashtags. So, a future area of research would be to look more broadly at the concepts that users are employing in their tweets. So, instead of just clustering on hashtags, one could look at clustering on hashtags and topical labels from something like the tweet text. Second, for the multi-view clustering of large scale clustering of hashtags in social media data there is a need for future research on the appropriate views and how to feature engineer those views to be useful. The URLs view of the data ended up being unhelpful for the found clusters, and this seems to be due in large part to the fact that there was very little overlap on exact URLs. So, there were situations in which essentially the same story or piece of news was used with two different hashtags in two different tweets, but because the URLs were not exactly the same, those hashtags were not recognized as being similar by the method. So, a means of processing the URLs to do something like just using the top level domains should be tried in future work. Additionally, previous research on misinformation during the COVID-19 pandemic has demonstrated that it can spread quickly by mechanisms like retweeting. It would be of value to create a tweet type view to characterize what type of tweets are being used with certain hashtags. Such a view may help with distinguishing between clusters of hashtags used for misinformation versus those used for more legitimate information. Second, there is also potential for future research in better cleaning and representing realworld data for multi-view clustering. For example, the tweets used in this study were not filtered by language. Performing a filtering step like only using English-language tweets could lead to more nuanced and meaningful clusters. Also, we adopted a heuristic graph learning procedure to form the view graphs for multi-view clustering of this data due to scalability issues with many of the more sophisticated graph learning procedures. Thus, an important avenue for future research is to find a graph learning procedure for MVMC that can better fit the intrinsic structure of the data, but that is also scalable to hundreds of thousands of entities. Finally, the data used in this study was only a sample of the twitter data pertaining to COVID-19. It would be interesting to see if different COVID-19 
twitter data yield the same results and if there are differences between different social media platforms that also employ hashtags.

\section{Abbreviations}

MVMC: Multi-view modularity clustering; kNN: k-Nearest neighbor, URL: Uniform resource locator

\section{Authors' contributions}

lain Cruickshank and Kathleen M. Carley have developed together the theoretical setup of the study. lain Cruickshank created the algorithmic framework, conducted the quantitative analyses and wrote the paper. Kathleen M. Carley has supervised the entire project. The author(s) read and approved the final manuscript.

\section{Funding}

This material is based upon work supported by the National Science Foundation Graduate Research Fellowship (DGE 1745016), Department of Defense Minerva Initiative (N00014-15-1-2797), and Office of Naval Research Multidisciplinary University Research Initiative (N00014-17-1-2675). Any opinion, findings, and conclusions or recommendations expressed in this material are those of the authors and do not necessarily reflect the views of the National Science Foundation, Department of Defense, or the Office of Naval Research.

\section{Availability of data and materials}

The datasets used and/or analysed during the current study are available from the corresponding author, or CASOS institute at casos@cmu.edu on reasonable request. Python implementations of the methods presented in this study are available from the corresponding author on reasonable request.

\section{Ethics approval and consent to participate}

Not applicable.

\section{Competing interests}

The authors declare that they have no competing interests.

Received: 1 July 2020 Accepted: 8 September 2020

Published online: 16 September 2020

\section{References}

Aleta A, Moreno Y (2019) Multilayer networks in a nutshell. Ann Rev Condens Matter Phys 10:45-62. https://doi.org/10. 1146/annurev-conmatphys-031218-013259 http://arxiv.org/abs/1804.03488

Alinezhad E, Teimourpour B, Sepehri MM, Kargari M (2019) Community detection in attributed networks considering both structural and attribute similarities: two mathematical programming approaches. Neural Comput \& Applic 32(8):3203-3220. https://doi.org/10.1007s00521-019-04064-5

Article1 9 (2020) Viral lies: Misinformation and the coronavirus. Technical report. https://www.article19.org/wp-content/ uploads/2020/03/Coronavirus-briefing.pdf. Accessed 1 Apr 2020

Bai S, Sun S, Bai X, Zhang Z, Tian Q (2018) Improving context-sensitive similarity via smooth neighborhood for object retrieval. Pattern Recog 83:353-364. https://doi.org/10.1016/j.patcog.2018.06.001

Baltrusaitis T, Ahuja C, Morency L (2017) Multimodal machine learning: A survey and taxonomy. CoRR abs/1705.09406:423-443. http://arxiv.org/abs/1705.09406

Baltrušaitis T, Ahuja C, Morency L (2019) Multimodal machine learning: A survey and taxonomy. IEEE Tran Pattern Anal Mach Intell 41(2):423-443. https://doi.org/10.1109/TPAMI.2018.2798607

Blondel VD, Guillaume J-L, Lambiotte R, Lefebvre E (2008) Fast unfolding of communities in large networks. J Stat Mech Theory Exp 2008(10):10008. https://doi.org/10.1088/1742-5468/2008/10/P10008 http://arxiv.org/abs/0803.0476

Boberg S, Quandt T, Schatto-Eckrodt T, Frischlich L (2020) Pandemic populism: facebook pages of alternative news media and the corona crisis - a computational content analysis. arXiv e-prints:2004-02566. http://arxiv.org/abs/2004.02566

Boongoen T, lam-On N (2018) Cluster ensembles: A survey of approaches with recent extensions and applications. Comput Sci Rev 28:1-25. https://doi.org/10.1016/j.cosrev.2018.01.003

Brugere I, Gallagher B, Berger-Wolf TY (2018) Network structure inference, a survey: Motivations, methods, and applications. ACM Comput Surv 51(2):24-12439. https://doi.org/10.1145/3154524

Chunaev P (2019) Community detection in node-attributed social networks: a survey. arXiv e-prints 37:1912-09816 http://arxiv.org/abs/1912.09816

Cinelli M, Quattrociocchi W, Galeazzi Ar, Valensise CM, Brugnoli E, Schmidt AL, Zola P, Zollo F, Scala A (2020) The COVID-19 social media infodemic. arXiv e-prints:2003-05004. http://arxiv.org/abs/2003.05004

Combe D, Largeron C, Géry M, Egyed-Zsigmond E (2015) I-louvain: An attributed graph clustering method. In: Fromont E, De Bie T, van Leeuwen M (eds). Advances in Intelligent Data Analysis XIV. Springer, Cham. pp 181-192

Corporation BB (2020) Li wenliang: Coronavirus kills chinese whistleblower doctor. BBC News

Cruickshank IJ (2020) Multi-view clustering of social-based data. PhD thesis, Carnegie Mellon University

de Santiago R, Lamb LC (2020) A ground truth contest between modularity maximization and modularity density maximization. Artif Intell Rev 53(6):4575-4599. https://doi.org/10.1007/s10462-019-09802-856789

Fern XZ, Brodley CE (2004) Solving cluster ensemble problems by bipartite graph partitioning. In: Proceedings of the Twenty-first International Conference on Machine Learning. ICML '04. ACM, New York, NY, USA. p 36. https://doi.org/ $10.1145 / 1015330.1015414$

Ferrara E (2020) \#COVID-19 on twitter: bots, conspiracies, and social media activism. arXiv e-prints:2004-09531. http://arxiv.org/abs/2004.09531 
Fortunato S, Barthelemy M (2007) Resolution limit in community detection. Proc Natl Acad Sci 104(1):36-41. https://doi.org/10.1073/pnas.0605965104 http://arxiv.org/abs/physics/0607100

Gallotti R, Valle F, Castaldo N, Sacco P, De Domenico M (2020) Assessing the risks of "infodemics" in response to COVID-19 epidemics. arXiv e-prints,:2004-03997. http://arxiv.org/abs/2004.03997

Guo D, Zhang J, Liu X, Cui Y, Zhao C (2014) Multiple kernel learning based multi-view spectral clustering. In: 2014 22nd International Conference on Pattern Recognition. IEEE, Stockholm. pp 3774-3779. https://doi.org/10.1109//CPR.2014. 648

Huang B (2020) Learning user latent attributes on social media. PhD thesis, Carnegie Mellon University

Huang S, Chaudhary K, Garmire LX (2017) More is better: Recent progress in multi-omics data integration methods. Front Genet 8:84. https://doi.org/10.3389/fgene.2017.00084

Huang Y, Panahi A, Krim H, Dai L (2019) Community detection and improved detectability in multiplex networks. arXiv e-prints:1909-10477. http://arxiv.org/abs/1909.10477

Hubert L, Arabie P (1985) Comparing partitions. J Classif 2(1):193-218. https://doi.org/10.1007/BF01908075

Hussain W (2020) The International Journal of Frontier Sciences. Role of social media in covid-19 pandemic 4:59-60. https://doi.org/10.37978/tijfs.v4i2.144

Kywe SM, Hoang T-A, Lim E-P, Zhu F (2012) On recommending hashtags in twitter networks. In: Proceedings of the 4th International Conference on Social Informatics. SocInfo'12. Springer, Berlin, Heidelberg. pp 337-350. https://doi.org/ 10.1007/978-3-642-35386-4_25

Lancichinetti A, Fortunato S (2011) Limits of modularity maximization in community detection. Physical Review E 84:066122. https://doi.org/10.1103/PhysRevE.84.066122 http://arxiv.org/abs/1107.1155

Magelinski T, Carley KM (2019) Community-based time segmentation from network snapshots: Streaming and holistic approaches for semi-static and dynamic nodesets. Appl Netw Sci 4(25):1-19. https://doi.org/10.1007/s41109-0190136-1

Maier M, Hein M, von Luxburg U (2009) Optimal construction of k-nearest neighbor graphs for identifying noisy clusters. arXiv e-prints 410(19):0912-3408. http://arxiv.org/abs/0912.3408

Maier M, von Luxburg U, Hein M (2011) How the result of graph clustering methods depends on the construction of the graph. arXiv e-prints 17:1102-2075. http://arxiv.org/abs/1102.2075

Masuda N, Holme P (2019) Detecting sequences of system states in temporal networks. Sci Rep 9(795):1-11. https://doi. org/0.1038/s41598-018-37534-2

Mucha PJ, Richardson T, Macon K, Porter MA, Onnela J-P (2010) Community structure in time-dependent, multiscale, and multiplex networks. Science 328(5980):876-878. https://doi.org/10.1126/science.1184819 https://science. sciencemag.org/content/328/5980/876.full.pdf

Newman MEJ (2010) Networks: an introduction. Oxford University Press, New York

Newman MEJ (2016) Community detection in networks: Modularity optimization and maximum likelihood are equivalent. arXiv e-prints 94:1606-02319. http://arxiv.org/abs/1606.02319

$\mathrm{Ni}$ J, Tong H, Fan W, Zhang X (2015) Flexible and robust multi-network clustering. In: Proceedings of the 21th ACM SIGKDD International Conference on Knowledge Discovery and Data Mining. KDD '15. Association for Computing Machinery, New York, NY, USA. pp 835-844. https://doi.org/10.1145/2783258.2783262

Pamfil AR, Howison SD, Lambiotte R, Porter MA (2018) Relating modularity maximization and stochastic block models in multilayer networks. CoRR abs/1804.01964:667-698. http://arxiv.org/abs/1804.01964

Papadopoulos A, Pallis G, Dikaiakos MD (2017) Weighted clustering of attributed multi-graphs. Computing 99:813-840

Papadopoulos A, Rafailidis D, Pallis G, Dikaiakos MD (2015) Clustering attributed multi-graphs with information ranking. In: Chen Q, Hameurlain A, Toumani F, Wagner R, Decker H (eds). Database and Expert Systems Applications. Springer, Cham. pp 432-446

Qiao L, Zhang L, Chen S, Shen D (2018) Data-driven graph construction and graph learning: A review. Neurocomputing 312:336-351. https://doi.org/10.1016/j.neucom.2018.05.084

Reichardt J, Bornholdt S (2006) Statistical mechanics of community detection. Phys Rev E 74:016110. https://doi.org/10. 1103/PhysRevE.74.016110

Saxton GD, Niyirora JN, Guo C, Waters RD (2015) \#advocatingforchange: The strategic use of hashtags in social media advocacy. Adv Soc Work 16(1):154-169. https://doi.org/10.18060/17952

Shapp A (2014) Variation in the use of twitter hashtags. Master's thesis, New York University. https://www.nyu.edu/ projects/shapp/Shapp_QP2_Hashtags_Final.pdf

Sheldon P, Herzfeldt E, Rauschnabel PA (2019) Culture and social media: the relationship between cultural values and hashtagging styles. Behav Inform Technol 0(0):1-13. https://doi.org/10.1080/0144929X.2019.1611923

Tagarelli A, Amelio A, Gullo F (2017) Ensemble-based community detection in multilayer networks. Data Min Knowl Disc 31:1506-1543

Traag VA, Krings G, van Dooren P (2013) Significant scales in community structure. Sci Rep 3:1-9

Traag VA, van Dooren P, Nesterov Y (2011) Narrow scope for resolution-limit-free community detection. Phys Rev E 84(1):016114. https://doi.org/10.1103/PhysRevE.84.016114

Traag VA, Waltman L, van Eck NJ (2019) From louvain to leiden: guaranteeing well-connected communities. Nat Sci Rep 9:1-12. https://doi.org/10.1038/s41598-019-41695-z

Vicient C, Moreno A (2015) Unsupervised topic discovery in micro-blogging networks. Expert Syst Appl 42(17):6472-6485. https://doi.org/10.1016/j.eswa.2015.04.014

Xia R, Pan Y, Du L, Yin J (2014) Robust multi-view spectral clustering via low-rank and sparse decomposition. In: AAAI Press, Quebec

Xiao F, Noro T, Tokuda T (2014) Finding news-topic oriented influential twitter users based on topic related hashtag community detection. J Web Eng 13:405-429

Xu Z, Ke Y (2016) Effective and efficient spectral clustering on text and link data. In: Proceedings of the 25th ACM International on Conference on Information and Knowledge Management. CIKM '16. Association for Computing Machinery, New York, NY, USA. pp 357-366. https://doi.org/10.1145/2983323.2983708 
Yang K-C, Torres-Lugo C, Menczer F (2020) Prevalence of Low-Credibility Information on Twitter During the COVID-19 Outbreak. arXiv e-prints:2004-14484. http://arxiv.org/abs/2004.14484

Yang Y, Wang H (2018) Multi-view clustering: A survey. Big Min Analytics 1(2):83-107

Ye F, Chen Z, Qian H, Li R, Chen C, Zheng Z (2018) New approaches in multi-view clustering. Recent Appl Data Clustering 11:195-220. https://doi.org/10.5772/intechopen.75598

Yu H, Lian Y, Zong L, Tian L (2017) Self-paced learning based multi-view spectral clustering. In: 2017 IEEE 29th International Conference on Tools with Artificial Intelligence (ICTAI). IEEE, Boston. pp 6-10. https://doi.org/10.1109/ ICTAI.2017.00013

Zhang Y (2019) Language in our time: An empirical analysis of hashtags. In: The World Wide Web Conference. WWW '19. Association for Computing Machinery, New York, NY, USA. pp 2378-2389. https://doi.org/10.1145/3308558.3313480

Zhu X, Loy CC, Gong S (2014) Constructing robust affinity graphs for spectral clustering. In: 2014 IEEE Conference on Computer Vision and Pattern Recognition. IEEE, Columbus. pp 1450-1457. https://doi.org/10.1109/CVPR.2014.188

Zitnik M, Nguyen F, Wang B, Leskovec J, Goldenberg A, Hoffman MM (2018) Machine learning for integrating data in biology and medicine: principles, practice, and opportunities. arXiv e-prints 50:1807-00123. http://arxiv.org/abs/ 1807.00123

\section{Publisher's Note}

Springer Nature remains neutral with regard to jurisdictional claims in published maps and institutional affiliations.

\section{Submit your manuscript to a SpringerOpen ${ }^{\circ}$} journal and benefit from:

- Convenient online submission

- Rigorous peer review

- Open access: articles freely available online

- High visibility within the field

- Retaining the copyright to your article

Submit your next manuscript at $\gg$ springeropen.com 